\title{
Integrated Effects of Genetic Resistance and Prothioconazole + Tebuconazole Application Timing on Fusarium Head Blight in Wheat
}

P. A. Paul ${ }^{\dagger}$ and J. D. Salgado, Department of Plant Pathology, The Ohio State University, Ohio Agricultural Research and Development Center, Wooster, OH 44691; G. Bergstrom, Plant Pathology and Plant-Microbe Biology Section, School of Integrative Plant Science, Cornell University, Ithaca, NY 14853; C. A. Bradley, Department of Plant Pathology, University of Kentucky Research and Education Center, Princeton, KY 42445; E. Byamukama, South Dakota State University, Department of Agronomy, Horticulture, and Plant Sciences, Brookings, SD 57007; A. M. Byrne, Department of Plant, Soil and Microbial Sciences, Michigan State University, East Lansing, MI 48824; V. Chapara, North Dakota State University, Langdon Research Extension Center, Langdon, ND 58249; J. A. Cummings, Plant Pathology and PlantMicrobe Biology Section, School of Integrative Plant Science, Cornell University, Ithaca, NY 14853; M. I. Chilvers, Department of Plant, Soil and Microbial Sciences, Michigan State University, East Lansing, MI 48824; R. Dill-Macky, Department of Plant Pathology, University of Minnesota, Saint Paul, MN 55108; A. Friskop, North Dakota State University, Department of Plant Pathology, Fargo, ND 58102; N. Kleczewski, Department of Plant and Soil Sciences, The University of Delaware, Newark, DE 19719; L. V. Madden, Department of Plant Pathology, The Ohio State University, Ohio Agricultural Research and Development Center, Wooster, OH 44691; M. Nagelkirk, Michigan State University Extension, Sandusky, MI 48471; J. Stevens, University of Nebraska-Lincoln, Department of Plant Pathology, NE 68538; M. Smith, Department of Plant Pathology, University of Minnesota, Northwest Research and Outreach Center, Crookston, MN 56716; S. N. Wegulo, University of Nebraska-Lincoln, Department of Plant Pathology, NE 68538; and K. Wise, Department of Plant Pathology, University of Kentucky Research and Education Center, Princeton, KY 42445; and D. Yabwalo, South Dakota State University, Department of Agronomy, Horticulture, and Plant Science, Brookings, SD 57007

\begin{abstract}
Integrated Fusarium head blight (FHB) management programs consisting of different combinations of cultivar resistance class and an application of the fungicide prothioconazole + tebuconazole at or after $50 \%$ early anthesis were evaluated for efficacy against FHB incidence (INC; percentage of diseased spikes), index (IND; percentage of diseased spikelets per spike), Fusarium damaged kernel (FDK), deoxynivalenol (DON) toxin contamination, grain yield, and test weight (TW) in inoculated field trials conducted in 11 U.S. states in 2014 and 2015. Mean log response ratios and corresponding percent control values for INC, IND, FDK, and DON, and mean differences in yield and TW relative to a nontreated, inoculated susceptible check (S_CK), were estimated through network meta-analyses as measures of efficacy. Results from the analyses were then used to estimate the economic benefit of each management program for a range of grain prices and fungicide applications costs. Management programs consisting of a moderately resistant (MR) cultivar treated with the fungicide were the most efficacious, reducing INC by 60 to $69 \%$, IND by 71 to $76 \%$, FDK by 66 to $72 \%$, and DON by 60 to $64 \%$ relative to S_CK, compared with 56 to $62 \%$ for INC, 68 to $72 \%$ for IND, 66 to

$68 \%$ for FDK, and 58 to $61 \%$ for DON for programs with a moderately susceptible (MS) cultivar. The least efficacious programs were those with a fungicide application to a susceptible (S) cultivar, with less than a $45 \%$ reduction of INC, IND, FDK, or DON. All programs were more efficacious under conditions favorable for FHB compared with less favorable conditions, with applications made at $50 \%$ early anthesis being of comparable efficacy to those made 2 to 7 days later. Programs with an MS cultivar resulted in the highest mean yield increases relative to S_CK (541 to $753 \mathrm{~kg} / \mathrm{ha}$ ), followed by programs with an $\mathrm{S}$ cultivar (386 to $498 \mathrm{~kg} / \mathrm{ha}$ ) and programs with an MR cultivar (250 to $337 \mathrm{~kg} / \mathrm{ha}$ ). Integrated management programs with an MS or MR cultivar treated with the fungicide at or after $50 \%$ early anthesis were the most likely to result in a 50 or $75 \%$ control of IND, FDK, or DON in a future trial. At a fixed fungicide application cost, these programs were $\$ 4$ to $\$ 319 / \mathrm{MT}$ more economically beneficial than corresponding fungicide-only programs, depending on the cultivar and grain price. These findings demonstrate the benefits of combining genetic resistance with a prothioconazole + tebuconazole treatment to manage FHB, even if that treatment is applied a few days after $50 \%$ early anthesis.
\end{abstract}

Fusarium head blight (FHB), incited predominantly by the fungus Fusarium graminearum in North America, and its associated mycotoxins, especially deoxynivalenol (DON), cause substantial grain

${ }^{\dagger}$ Corresponding author: P. A. Paul; E-mail: paul.661@ osu.edu

Funding: This is a cooperative project with the U.S. Wheat \& Barley Scab Initiative (Agreements \# 59-0206-4-018 [The Ohio State University], 590206-4-006 [Cornell University], 59-0206-4-042 [Purdue University], 590206-4-016 [The University of Minnesota], 59-0206-4-037 [Michigan State University], 59-0206-4-024 [University of Illinois], 59-0206-4-036 [The University of Delaware], 59-0206-4-012 [North Dakota State University], 590206-4-005 [South Dakota State University], and 59-0206-2-085 [University of Nebraska-Lincoln]). Any opinions, findings, conclusions, or recommendations expressed in this publication are those of the authors and do not necessarily reflect the view of the U.S. Department of Agriculture. Salaries and research support for P. A. Paul, L. V. Madden, and J. D. Salgado were provided by state and federal funds to the Ohio Agricultural Research and Development Center.

Accepted for publication 18 July 2018.

(c) 2019 The American Phytopathological Society yield and quality losses in all classes of wheat (McMullen et al. 1997, 2012). Considerable progress has been made over the last 15 years to develop strategies to minimize such losses. Several cultivars with improved resistance have been developed and released, efficacious fungicides have been evaluated and registered for use against FHB, disease forecasting models have been developed and deployed to help guide fungicide applications, and the value of combining multiple strategies to manage this disease-toxin complex has been demonstrated (McMullen et al. 2008, 2012). Results from several years of coordinated integrated management trials conducted in 12 U.S. states showed that relative to the nontreated susceptible check, the combination of a moderately resistant cultivar and a prothioconazole + tebuconazole (Prosaro; Bayer CropScience, Research Triangle Park, NC) application at early anthesis (Feekes 10.5.1) resulted in more than $70 \%$ control of both FHB index and DON. On average, the integration of these two management strategies was $21 \%$ more effective than moderate cultivar resistance or a fungicide application alone for index, and $21 \%$ more effective than moderate cultivar resistance alone and $31 \%$ more effective than fungicide alone for DON (Willyerd et al. 2012).

In spite of the progress, regional FHB epidemics have occurred periodically in parts of the Midwest, Mid-South, Southeast, and Great 
Plains during the last decade (McMullen et al. 2012), indicating that there is still work to be done to improve FHB management and minimize losses due to the disease. It is clear from previous studies that the integration of multiple strategies, including fungicide applications, genetic resistance, rotation with non-host crops, and infield residue management, provides the best FHB and DON control (McMullen et al. 2008; Mesterházy et al. 2003; Wegulo et al. 2011; Willyerd et al. 2012). However, practical limitations often prevent producers and crop advisors from following management recommendations. For instance, although metconazole (Caramba; BASF Corp., Research Triangle Park, NC) and tebuconazole + prothioconazole are rain-fast within an hour after application (Andersen et al. 2014; Mills et al. 2017), wet field conditions may make it difficult, if not impossible, for ground applications to be made at the recommended growth stage of early anthesis. Other limitations to adequately time fungicide applications include uneven crop development within a field, a variable anthesis window as influenced by cultivar characteristics and weather conditions, difficulties in scheduling fungicide applicators, and the inability to correctly determine if the crop is at anthesis. These limitations led to the development of a series of coordinated uniform fungicide trials (UFTs) and integrated management coordinated projects (IMCP) through the U.S. Wheat and Barley Scab Initiative (USWBSI) to evaluate and develop robust fungicide application guidelines that support their use under conditions experienced in the field.

Results from UFTs designed to investigate the effects of pre- and post-anthesis fungicide applications on FHB and DON showed that an application made between 2 and 6 days after Feekes 10.5.1 (referred to here as post-anthesis) was just as effective against DON as an application made at Feekes 10.5.1 (Paul et al. 2018). In fact, results from studies conducted in Ohio and Illinois in which wheat was naturally infected as well as spawn- and spray-inoculated showed that in some cases, postanthesis fungicide treatments provided numerically greater DON reductions than fungicide treatments applied at early anthesis (Bradley et al. 2009, 2010; D'Angelo et al. 2014). In a study encompassing 16 states over 19 years, Paul et al. (2018) reported that the efficacy of postanthesis (curative) applications of tebuconazole + prothioconazole or metconazole was greater than pre-anthesis (preventative) applications. They also noted that efficacy was consistent between spring and winter wheat production regions in terms of percent FHB index control relative to a nontreated check, but greater in spring than in winter wheat regions in terms of percent DON reduction.

Building on the results from the UFTs in which pre- and postanthesis treatment effects were evaluated on susceptible cultivars, a series of USWBSI IMCP trials were conducted to evaluate postanthesis treatments as part of integrated management programs involving moderately susceptible and moderately resistant cultivars. Given that the baseline levels of FHB and DON vary among environments and cultivars with different levels of resistance, and that fungicide efficacy may vary with baseline FHB levels (Paul et al. 2007, 2008), the IMCP data were invaluable for determining whether the efficacy of late applications was influenced by cultivar resistance and baseline disease levels. Here we present findings from network meta-analyses (Madden et al. 2016) of data from a group of 29 IMCP trials to quantify: 1) the effect of integrating a post-anthesis application of prothioconazole + tebuconazole and genetic resistance on FHB incidence, a measure of infection, and FHB index (field severity), a measure of infection and spread of the fungus within the spike; 2 ) the effect of different genetic resistance by fungicide (specifically prothioconazole + tebuconazole) management combinations on grain yield and quality traits such as percent Fusarium damaged kernels (FDK), DON contamination of harvested grain, and test weight; 3 ) the influence of baseline FHB index, a surrogate measure of environmental favorability for FHB development, on the efficacy of prothioconazole + tebuconazole by genetic resistance combinations against FHB index, FDK, and DON; and 4) the economic benefit of prothioconazole + tebuconazole by genetic resistance combinations.

\section{Materials and Methods}

FHB integrated management trials. Coordinated integrated management trials were conducted during the 2013-14 and
2014-15 spring and winter wheat growing seasons in 11 U.S. states to evaluate the effects of cultivar resistance by fungicide treatment combinations on FHB incidence (INC) and index (IND), FDK, DON, grain yield, and test weight. Trials were established in 29 environments, representing unique combinations of years and locations (Table 1). In all cases, a standard set of protocols in terms of experimental design, treatment application, and data collection was used. Either soft red winter wheat (SRWW), hard red spring wheat (HRSW), hard red winter wheat (HRWW), or soft white winter wheat (SWWW) cultivars with different levels of type II resistance (Bal and Shaner 1994, 2004; Schroeder and Christensen 1963) to FHB (Table 1; moderately resistant [MR], moderately susceptible [MS], and susceptible [S]) were planted as whole-plots in a randomized complete block design with four to six replicate blocks. Plots were established and managed according to standard production practices for each location (Barker et al. 2017; Conley et al. 2014; Mansfield and Hawkins 1992; Nafziger 2009) either on university research farms or in commercial fields planted the previous growing season with corn, oats, wheat, dry bean, red clover, or soybean.

Plots of each cultivar were divided into subplots with dimensions ranging in width from 1.5 to $3 \mathrm{~m}$ and length from 6 to $10.7 \mathrm{~m}$, to which separate fungicide treatments were applied. Only the four most widely used treatments across the 29 environments, plus a nontreated check, were included in the analysis presented in this study. The four treatments consisted of the demethylation inhibitor fungicides prothioconazole + tebuconazole (Prosaro; Bayer CropScience, Research Triangle Park, NC) applied at a rate of $475 \mathrm{ml}$ per hectare (this corresponds to approximately $100 \mathrm{~g}$ of each active ingredient per hectare) at $50 \%$ early anthesis (Feekes growth stage 10.5.1), 2 to 3 days after $50 \%$ early anthesis, 4 to 5 days after $50 \%$ early anthesis, or 6 to 7 days after $50 \%$ early anthesis. Henceforth, the early anthesis treatment will be abbreviated as $\mathrm{A}$, whereas the post-anthesis treatments will be abbreviated as $A+2, A+4$, and $A+6$, respectively. All treatments were applied along with a nonionic surfactant at a concentration of $0.125 \% \mathrm{v} / \mathrm{v}$ and a spray volume of 197 liters ha ${ }^{-1}$. Applications were made using backpack or tractor-mounted sprayers fitted with paired Twinjet or flat fan XR8001 nozzles, mounted at an angle ( 30 to $45^{\circ}$ from the horizontal) forward and backward or forward only.

$F$. graminearum infection and FHB development were enhanced by one or more of the following: planting after an $F$. graminearumsusceptible host crop (corn or wheat), artificial inoculation with $F$. graminearum-colonized grain spawn, or spray-inoculation with a spore suspension $\left(40 \times 10^{3}\right.$ to $100 \times 10^{3}$ spores $\left./ \mathrm{ml}\right)$ of $F$. graminearum between 24 and $36 \mathrm{~h}$ after the early anthesis treatments were applied (Table 1). In some cases, experiments were mist-irrigated during anthesis and early grain-fill to promote disease development. FHB INC (mean percentage of diseased spikes) and IND (mean percentage of diseased spikelets per spike) (Paul et al. 2005; Stack and McMullen 1998) were quantified on 50 to 100 spikes per plot at the soft dough growth stage (Feekes 11.2), approximately 18 to 21 days after anthesis. Plots were harvested using research plot combines, and grain yield and test weight (adjusted to $13.5 \%$ moisture) were estimated and converted to kilograms per hectare $(\mathrm{kg} / \mathrm{ha})$ and kilograms per cubic meter $\left(\mathrm{kg} / \mathrm{m}^{3}\right)$, respectively. Grain samples from each subplot were rated for percent FDK (percentage of visibly diseased [shriveled, pinkish-white, and lightweight] kernels) and assayed for DON at a USWBSI testing laboratory.

Data analysis. Least squares means and standard errors. The data were first analyzed by fitting separate linear mixed models to each response in each trial. Models were fitted using the GLIMMIX procedure of SAS (Littell et al. 2006), with treatment and cultivar as fixed effects and block as a random effect. The lsmeans statement in GLIMMIX was then used to obtain estimates of least squares means and standard errors for each combination of fungicide treatment (application timing) and cultivar resistance class. Across the 29 trials, means and standard errors were estimated for a total of 15 cultivar resistance class by fungicide treatment combinations, namely: 1) MR A, MR treated with prothioconazole + tebuconazole at $50 \%$ early anthesis; 2) MR_A+2, MR treated at 2 to 3 days after $50 \%$ early 
anthesis; 3) MR_A+4, MR treated at 4 to 5 days $50 \%$ after early anthesis; 4) MR_A+6, MR treated at 6 to 7 days after $50 \%$ early anthesis; 5) MR_CK, MR nontreated; 6) MS_A, MS treated at 50\% early anthesis; 7) MS_A+2, MS treated at 2 to 3 days after $50 \%$ early anthesis; 8) MS_A+4, MS treated at 4 to 5 days after $50 \%$ early anthesis; 9) MS_A+6, MS treated at 6 to 7 days after $50 \%$ early anthesis; 10) MS_CK, MS nontreated; 11) S_A, S treated at 50\% early anthesis; 12) $S \_A+2, S$ treated at 2 to 3 days after $50 \%$ early anthesis; 13 ) S_A+4, S treated at 4 to 5 days after $50 \%$ early anthesis, 14) S_A+ $6, \mathrm{~S}$ treated at 6 to 7 days after $50 \%$ early anthesis; and 15) S_CK, $\mathrm{S}$ nontreated. For those trials with multiple cultivars in a given resistance class, means were estimated across all cultivar $\times$ treatment combinations for that particular resistant class. The number of trials in which each treatment $\times$ resistance class combination was evaluated for its effect on INC, IND, FDK, DON, grain yield, and test weight is shown in Figure 1.

Management combination effects on Fusarium head blight intensity and grain quality. Outputs from the linear mixed model analyses were used to generate a data matrix of least squares means and corresponding standard errors for network meta-analysis. For INC, IND, FDK, and DON, log response ratios (Hedges et al. 1999) and approximate percent controls were estimated as measures of efficacy of each resistance class by fungicide treatment management combination. Log of the response ratio $(L)$ for a single pair of treatments is calculated as:

$$
L=\ln (R)=\ln \left(X_{M g m t} / \bar{X}_{S_{-} C K}\right)=\ln \left(\bar{X}_{M g m t}\right)-\ln \left(\bar{X}_{S_{-} C K}\right)
$$

where $\bar{X}_{S_{-} C K}$ is the mean response for the susceptible nontreated check, used here as the reference management combination, and $\bar{X}_{M g m t}$ is the mean for one of the other 14 management combinations
(MR A, MR A+2, MR A+4, MR A+6, MR CK, MS A, MS A+2, MS_A+4, MS_A+6, MS_CK, S_A, S_A+2, S_A+4, and S_A+6).

Prior to fitting the meta-analytical model, mean INC, IND, FDK, and DON from each trial were log-transformed $\left[Y_{i j}=\ln \left(\bar{X}_{i j}\right)\right]$, and sampling variances of the log means for the $i$ th management combination in the $j$ th study were estimated as $s_{i j}^{2}=v_{j} / \bar{X}_{i j}^{2}$, where $v_{j}$ is the square of the standard error of the least squares means for the $j$ th study, $\bar{X}_{i j}$, as described by Willyerd et al. (2012). Network metaanalytical models were then fitted to the INC, IND, FDK, and DON data, with $\log$ of the response $\left(Y_{i j}\right)$ as the dependent variable and the inverse of the sampling variance as the fixed within-study weight $\left(w_{i j}=1 / s_{i j}^{2}\right)$, in order to estimate the overall expected log response ratios (across trials) of the different cultivar resistance class by fungicide treatment management combinations. Models were fitted to the data for each response variable using restricted maximum likelihood with the GLIMMIX procedure of SAS, as previously described (Madden et al. 2016; Paul et al. 2008; van Houwelingen et al. 2002). Mean log response ratios, together with their standard errors and confidence intervals, were then estimated using lsmestimate statements with the $c l$ option in PROC GLIMMIX. For a given management combination, the mean log ratio across trials is estimated as:

$$
\bar{L}=\bar{Y}_{M g m t}-\bar{Y}_{S_{-} C K}
$$

where $\bar{Y}_{M g m t}$ is the estimated least-squares mean log response (INC, IND, FDK, or DON) for each management combination and $\bar{Y}_{S_{-} C K}$ is the least-squares mean $\log$ response for the susceptible nontreated check (S_CK). Equation 2 gives the log ratio because the $\log$ of the ratio is equal to the difference in logs (see Equation 1). The standard normal test statistic $(Z)$ was used to determine whether the log response ratios between S_CK and other management combinations

Table 1. Environments (Env) consisting of different combinations of years, states, and locations within states in which Fusarium head blight (FHB) integrated

\begin{tabular}{|c|c|c|c|c|c|c|c|}
\hline Env & Year & State & Location & Cultivar (FHB resistance classification) ${ }^{\mathbf{a}}$ & Class $^{\mathbf{b}}$ & $\mathbf{P C}^{\mathbf{c}}$ & Inoc $^{d}$ \\
\hline 1 & 2014 & IL & Dixon Springs & BW5228 (MR), BW5530 (MR), P25R32 (MS), P25R47 (S) & SRWW & Corn & Spray \\
\hline 2 & 2014 & IL & Urbana & BW5228 (MR), BW5530 (MR), P25R32 (MS), P25R47 (S) & SRWW & Corn & Spray \\
\hline 3 & 2015 & IL & Dixon Springs & MW844 (MR), DG9441 (MS), P25R32 (MS), W1566 (S) & SRWW & Corn & Spray \\
\hline 4 & 2015 & IL & Urbana & MW844 (MR), DG9441 (MS), P25R32 (MS), W1566 (S) & SRWW & Corn & Spray \\
\hline 5 & 2014 & IN & Tippecanoe & Truman (MR), Malabar (MR), Bromfield (MS), Hopewell (S) & SRWW & Corn & Spray \\
\hline 6 & 2015 & IN & Tippecanoe & Truman (MR), Malabar (MR), Bromfield (MS), Hopewell (S) & SRWW & Corn & Spray \\
\hline 7 & 2014 & $\mathrm{OH}$ & Wooster & Truman (MR), Malabar (MR), Bromfield (MS), Hopewell (S) & SRWW & Oats & Spray \\
\hline 8 & 2015 & $\mathrm{OH}$ & Wooster & Truman (MR), Malabar (MR), Bromfield (MS), Hopewell (S) & SRWW & Oats & Spray \\
\hline 9 & 2014 & MI & Deckerville & Ambassador (S) & SWWW & Dry beans & Spray \\
\hline 10 & 2015 & MI & Deckerville & DG92W42 (MR), P25R34 (MR), Whale (MS), Ambassador (S) & SWWW & Dry beans & Spawn \\
\hline 11 & 2015 & MI & East Lansing & 9242W (MR), F1014 (MR), E6012 (MS), Ambassador (S) & SWWW & Red clover & Spawn \\
\hline 12 & 2014 & NY & Aurora & P25R46 (MR), Emmit (MS), Otsego (MS), P25R40 (S) & SRWW & Soybean & Spray \\
\hline 13 & 2015 & NY & Aurora & P25R46 (MR), Emmit (MS), Otsego (MS), P25R40 (S) & SRWW & Corn & Spray \\
\hline 14 & 2014 & $\mathrm{DE}$ & Georgetown & 25R32 (MR), 25R40 (S) & SRWW & Corn & Spray \\
\hline 15 & 2015 & $\mathrm{DE}$ & Georgetown & 25R50 (MR), 25R32 (MR), 25R40 (S), Shirley (S) & SRWW & Corn & Spray \\
\hline 16 & 2014 & MD & Wye & $12 \mathrm{~V} 51$ (MR), Merl (S) & SRWW & Corn & Spray \\
\hline 17 & 2014 & $\mathrm{NE}$ & Mead & Overland (MR), Everest (MR), McGill (S), Overley (S) & HRWW & Corn & Spawn/Spray \\
\hline 18 & 2015 & $\mathrm{NE}$ & Mead & Overland (MR), Everest (MR), McGill (S), Overley (S) & HRWW & Corn & Spawn/Spray \\
\hline 19 & 2014 & SD & Volga & Overland (MR), Alice (MS), Wesley (S) & HRWW & Wheat & Spawn \\
\hline 20 & 2014 & SD & Volga & Brick (MR), Prevail (MS), Samson (S) & HRSW & Corn & Spawn \\
\hline 21 & 2014 & SD & South Shore & Brick (MR), Prevail (MS), Samson (S) & HRSW & Corn & - \\
\hline 22 & 2015 & SD & Volga & Brick (MR), Prevail (MS), Samson (S) & HRSW & Corn & Spawn \\
\hline 23 & 2015 & SD & South Shore & Brick (MR), Prevail (MS), Samson (S) & HRSW & Corn & - \\
\hline 24 & 2015 & MN & Crookston & Linkert (MS), SYSoren (MS), Samson (S) & HRSW & Wheat & Spray \\
\hline 25 & 2015 & $\mathrm{MN}$ & St. Paul & Linkert (MS), SYSoren (MS), Samson (S) & HRSW & Soybean & Spray \\
\hline 26 & 2014 & ND & Fargo & Glenn (MR), Faller (MS), Briggs (S) & HRSW & Soybean & Spawn \\
\hline 27 & 2014 & ND & Langdon & Brick (MR), Faller (MS), Samson (S) & HRSW & Wheat & Spawn \\
\hline 28 & 2015 & ND & Fargo & Glenn (MR), Faller (MS), WB-Mayville (S) & HRSW & Corn & Spawn \\
\hline 29 & 2015 & ND & Langdon & Glenn (MR), Faller (MS), Samson (S) & HRSW & Canola & Spawn \\
\hline
\end{tabular}
management trials were conducted

${ }^{a}$ Resistance classes - moderately resistant (MR), moderately susceptible (MS), and susceptible (S) wheat cultivars.

${ }^{\mathrm{b}}$ Wheat market classes - soft red winter wheat (SRWW), soft white winter wheat (SWWW), hard red winter wheat (HRWW), and hard red spring wheat (HRSW). c Previous crop.

${ }^{\mathrm{d}}$ Spray $=$ inoculated at anthesis with a spore suspension of F. graminearum, spawn $=$ inoculated with $F$. graminearum-colonized grain spawn, and $-=$ without artificial inoculation. 
were statistically different from zero. Approximate mean percent control $(\bar{C})$ for INC, IND, FDK, and DON for each management combination relative to S_CK were calculated from the $\bar{L}$ values as $\bar{C}=[1-\exp (\bar{L})] \times 100$ (Madden and Paul 2011). Confidence intervals for percent control were likewise calculated from the upper and lower limits of the $95 \%$ confidence interval around $\bar{L}$.

Management combination effects on grain yield and test weight. For grain yield and test weight, mean differences were estimated as measures of management combination effects. The model fitting approach was similar to that described above for measures of FHB, with the primary difference being that the original least squares means from the linear mixed model analyses were used instead of their log transformation. Sampling variances and weights of the least squares means for the $i$ th management combination in the $j$ th study were estimated as $s_{i j}^{2}=v_{j}$ and $w_{i j}=1 / s_{i j}^{2}$, respectively, with $v_{j}$ again being the square of the standard error of least squares means from the linear mixed model fit to the original data. Overall mean differences $(\bar{D})$ across trials for each management combination relative
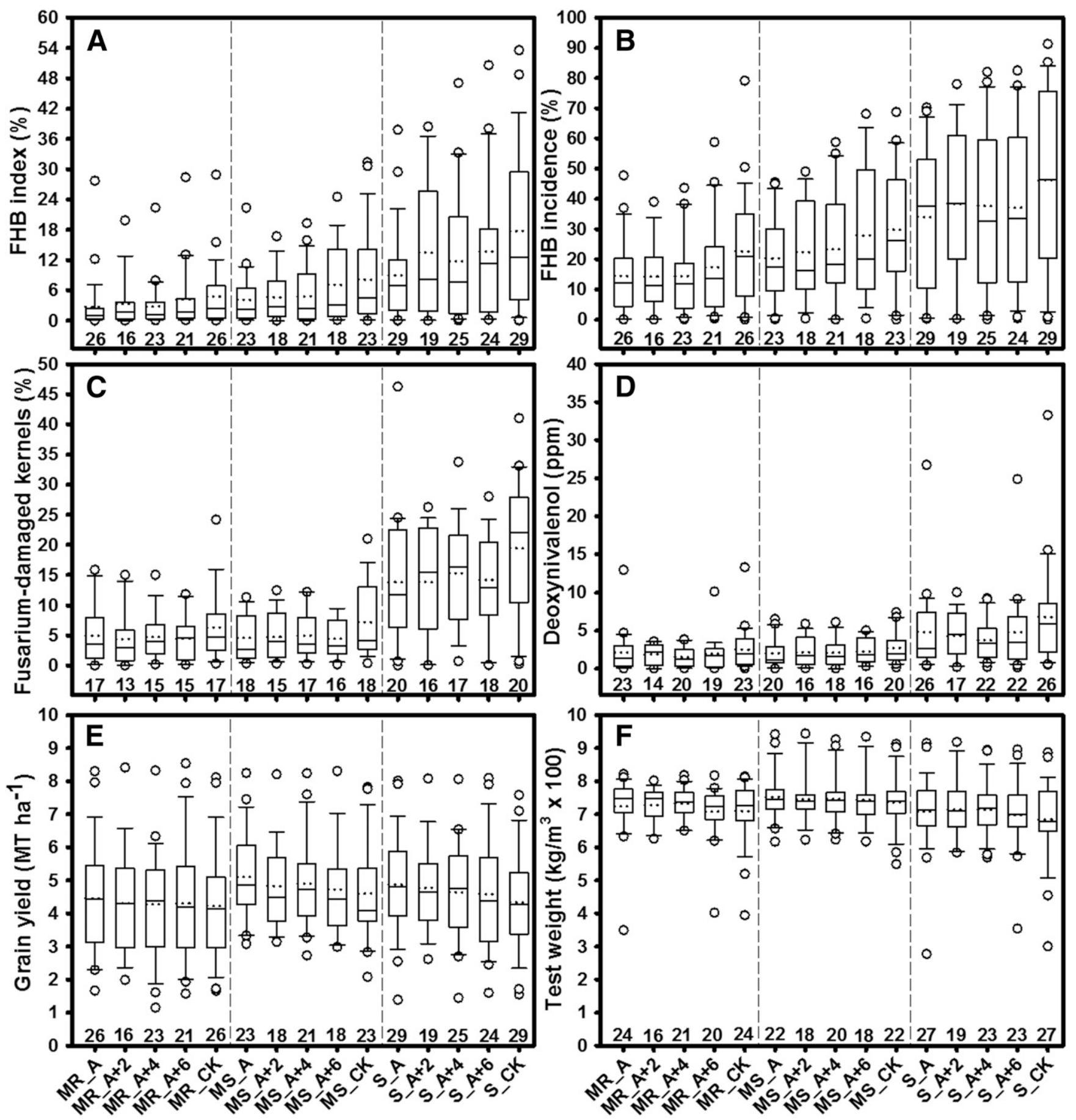

Fig. 1. Box plot of the distribution of least squares mean Fusarium head blight index (A) and incidence (B), Fusarium damaged kernels (C), deoxynivalenol content of harvested grain (D), grain yield (E), and test weight (F) for different fungicide treatment by cultivar resistance class management combinations. Combinations consisted of the application of the fungicide prothioconazole + tebuconazole at $50 \%$ early anthesis, or at 2,4 , or 6 days after $50 \%$ early anthesis $(A, A+2, A+4$, or $A+6$, respectively) to moderately resistant (MR), moderately susceptible (MS), or susceptible (S) wheat cultivars. Broken and solid lines within each box represent means and medians, respectively, while the top and bottom lines of the box represent the 75th and 25th percentiles of the data, respectively. Vertical bars extending beyond the boxes represent the 10th and 90th percentiles, while circles represent outliers. Numbers immediately above the x-axis indicate the number of trials in which each management combination was evaluated for its effect on each of the measured response. The vertical lines serve to facilitate comparisons among management combinations with S, MS, and MR cultivars. 
to S CK and their standard errors and $95 \%$ confidence intervals were estimated post network meta-analysis model fitting using the lsmestimate statement with the $c l$ option in PROC GLIMMIX. $\bar{D}$ was estimated as $\bar{Y}_{M g m t}-\bar{Y}_{S_{-} C K}$, where now $\bar{Y}_{M g m t}$ and $\bar{Y}_{S_{-} C K}$ are estimated least squares mean yield or test weight (across trials) for the management combination and nontreated susceptible check, respectively. See Paul et al. (2010) for details.

Influence of baseline FHB index on effect sizes. To determine whether the effects of management combinations varied with baseline levels of FHB, the network meta-analytical models were refitted to the IND, DON, FDK, grain yield, and test weight data, with mean IND in the nontreated susceptible check $\left(I_{N D}\right.$ S_CK $\left._{-}\right)$used as a categorical moderator variable and fixed effect. Based on $\mathrm{IND}_{\mathrm{S}_{\mathrm{CK}} \text {, trials }}$ were categorized into two groups, those with $\mathrm{IND}_{\mathrm{S}_{\mathrm{C}} \mathrm{CK}}>10 \%$ and those with $\mathrm{IND}_{\mathrm{S}_{-} \mathrm{CK}} \leq 10 \%$, high and low baseline levels of disease, respectively. This threshold, commonly used as a measure of the risk of an FHB epidemic (Shah et al. 2013, 2014), can be considered a surrogate measure of conditions favorable for FHB development. Trials with $\mathrm{IND}_{\mathrm{S} \_\mathrm{CK}}>10 \%$ were considered to be conducted under more favorable conditions for FHB than those with IND $_{\text {S_CK }_{-}} \leq 10 \%$. Models were fitted and effect sizes $(\bar{L}, \bar{C}$, and $\bar{D})$ and their standard errors and $95 \%$ confidence intervals estimated as described above and in Paul et al. $(2008,2010)$ for the analysis without moderator variable. However, instead of estimating a single effect size for each management combination, a pair of effect sizes corresponding to the two groups of trials (low and high IND $_{\mathrm{S}_{-} \mathrm{CK}}$ ) was estimated for each management combination. After fitting the models, lsmestimate statements in GLIMMIX were again used to estimate $\bar{L}$ for each management combination at each level of the moderator variable, and contrast statements were used to determine whether IND $_{\mathrm{S}_{-} \mathrm{CK}}$ had a significant effect on $\bar{L}$ for IND, DON, FDK, test weight, and grain yield based on $\chi^{2}$ tests.

Predicted efficacy and economic analysis. Using the estimated effect sizes and between-study variances $\left(\bar{L}\right.$ and $\left.\hat{\sigma}^{2}\right)$ from the metaanalyses, probabilities of achieving at least a $50 \%\left(p_{50}\right)$ and $75 \%$ $\left(p_{75}\right)$ IND, FDK, and DON control over S_CK in a new randomly selected trial, conducted in a manner similar to those described herein, were estimated for the 14 management combinations examined. For instance, $p_{50}$ for IND, FDK, or DON, equivalent to the probability that $\bar{L}<-0.69$, was estimated as $p_{50}=\varnothing\left((-0.69-\bar{L}) / \sqrt{\hat{\sigma}^{2}}\right)$, where $\varnothing(\bullet)$ is the cumulative standard-normal function (Madden and Paul 2011; Paul et al. 2008, 2010). Similarly, $p_{75}$, the probability of $\bar{L}<-1.39$, was estimated as $p_{75}=\varnothing\left((-1.39-\bar{L}) / \sqrt{\hat{\sigma}^{2}}\right)$. Probability values were estimated for both levels of the moderator variable, IND S_CK. $_{\text {. }}$

Effect sizes from the meta-analyses were also used, along with summary statistics (means and quartiles) from the distributions of within-trial least squares means across all trials (Fig. 1), grain prices, estimated price discounts, and fungicide application cost, to estimate net cash income $\left(\mathrm{NCI} ; \$ \mathrm{ha}^{-1}\right)$ as a measure of the economic benefit of each management combination. Details of this analysis can be found in Salgado et al. (2014, 2017). NCI was estimated for the nontreated susceptible check as

$$
N C I_{S_{-} C K}=Y l d \cdot(P-d c t)
$$

and for each management combination as

$$
N C I_{M g m t}=Y l d * \cdot(P-d c t *)-C_{0 s} t_{p}
$$

Here, $Y l d$ is estimated overall least squares mean (averaged across trials) grain yield for $\mathrm{S} \_\mathrm{CK}$ (metric tons [MT] ha $\mathrm{ha}^{-1}$ ); $P$ is standard price per MT paid by the grain elevator (US\$ $\mathrm{MT}^{-1}$ ); $d c t$ is the total price discount for S_CK (US\$ $\mathrm{MT}^{-1}$ ) based on grain quality (test weight; FDK, and DON) thresholds; $Y l d^{*}$ is the adjusted least squares mean yield $(Y l d+\bar{D})$ based on effect sizes from the metaanalysis; $d c t^{*}$ is the total price discount after adjusting for the effect of management combination ( $\bar{D}$ and $\bar{C}$ values) on test weight, FDK, and DON; and Cost $_{p}$ is production costs (fungicide application) directly related to FHB management (US\$/ha).
Using a slightly modified version of the example price discount schedule in Salgado et al. (2011), total price discount (dct) was estimated as $d c t=t w l+f d k l+d o n l$, where $t w l, f d k l$, and $d o n l$ represent individual price discounts due to test weight below $746.6 \mathrm{~kg} / \mathrm{m}^{3}$ (58 $\mathrm{lb}$ per bushel), $\mathrm{FDK} \geq 1 \%$, and $\mathrm{DON} \geq 2 \mathrm{ppm}$, respectively. For each $6.4 \mathrm{~kg} / \mathrm{m}^{3}$ (0.5 lb. per bushel) reduction in test weight, a price discount of US\$1.6/MT was applied. For FDK, a discount of US\$2.4/ MT was applied for the first $0.5 \%$ increase above $1 \%$, plus an additional US $\$ 0.79 / \mathrm{MT}$ for each additional $0.25 \%$ increase up to $2 \%$, after which an additional discount of US\$0.59/MT was applied per $0.5 \%$ increase in FDK up to $4 \%$. Then for each $0.5 \%$ increase in FDK above $4 \%$, a discount of US $\$ 0.98 / \mathrm{MT}$ was applied. For DON, a discount of US\$1.97/MT was applied for $2 \mathrm{ppm}$, plus US $\$ 1.97 / \mathrm{MT}$ for each additional $0.5 \mathrm{ppm}$ increase above $2 \mathrm{ppm}$, up to $5 \mathrm{ppm}$, and then an additional US\$3.94/MT was applied for each additional $0.5 \mathrm{ppm}$ increase in DON above $5 \mathrm{ppm}$.

\section{Results}

Overall efficacy of management combinations against FHB intensity and grain quality. The distributions of estimated least squares means are shown in Figure 1. Mean IND ranged from 0 to $54 \%$ (Fig. 1A), mean INC from 0 to $91 \%$ (Fig. 1B), mean FDK from 0 to $41 \%$ (Fig. 1C), and mean DON from 0 to 33 ppm (Fig. 1D). Variability of the means across trials, as indicated by the interquartile ranges (IQR; difference between the 75th and 25th percentiles of the data), was lowest for those management combinations that consisted of a fungicide application to a moderately susceptible or moderately resistant cultivar and highest for those with a susceptible cultivar. IQR values were generally higher for in-field measures of FHB, particularly INC, than for postharvest measures of grain quality (Fig. 1A, B, C, and D).

Based on overall mean log responses $(\bar{L})$ from the meta-analyses, all management combinations reduced mean INC, IND, FDK, and DON relative to the nontreated susceptible check (S_CK); $\bar{L}$ values were all negative and significantly different from zero $(P<0.005$; Tables 2 and 3 ). This corresponded to positive percent control $(\bar{C})$ values (Fig. 2) for all management programs. However, the magnitude of the effect varied among responses and management combinations. For responses quantified in the field (18 to 21 days after early anthesis), $\bar{C}$ values were higher for IND than for INC (Fig. 2A and B). For instance, an application of prothioconazole + tebuconazole at or after $50 \%$ early anthesis to an MR cultivar resulted in 71 to $76 \%$ control of IND compared with 60 to $69 \%$ control of INC. The corresponding ranges of percent control for management combinations with an MS cultivar were 66 to $72 \%$ for IND and 56 to $62 \%$ for INC. Resistance alone (MR_CK) reduced mean INC and IND by 48 and $66 \%$, respectively, relative to the $S \_C K$. The least effective programs, with $<35 \%$ control of INC and $<46 \%$ control of IND, were those consisting of a prothioconazole + tebuconazole application to a susceptible cultivar. In general, applications made at 2 days after $50 \%$ early anthesis resulted in slightly higher $\bar{C}$ values than those made at anthesis or 4 to 7 days after $50 \%$ early anthesis (Fig. 2A and B).

Early anthesis and later treatments also showed comparable efficacy against grain quality traits (FDK and DON) quantified after harvest, particularly when applied to MS or MR cultivars. As was the case with INC and IND, management combinations that include an MR cultivar treated with prothioconazole + tebuconazole at or after $50 \%$ early anthesis resulted in the highest overall mean percent control of FDK (71 to $72 \%$ ) and DON (60 to 64\%) relative to S_CK (Fig. 2C and D). The efficacy of applications made to MS cultivars ranged from 66 to $68 \%$ for FDK and 58 to $61 \%$ for DON. MR (MR CK) and MS (MS_CK) alone were generally less effective than the integration of resistance and fungicide treatment, but considerably more effective than programs consisting of a fungicide treatment alone (prothioconazole + tebuconazole application to a $S$ cultivar). For the latter group of management programs, $\bar{C}$ values ranged from 20 to $28 \%$ for FDK and from 26 to $34 \%$ for DON (Fig. 2C and D). 
Management combination effects on grain yield and test weight. For grain yield, least squares means in the nontreated susceptible check (S_CK) ranged from 1.5 to $7.6 \mathrm{MT} / \mathrm{ha}$, with an overall mean of 4.3 MT/ha (Fig. 1E). Averaged across trials, means for management programs with an MR cultivar ranged from 4.2 to 4.5 MT/ha, compared with 4.6 to $5.1 \mathrm{MT} / \mathrm{ha}$ for programs with an MS cultivar and 4.6 to $4.9 \mathrm{MT} / \mathrm{ha}$ for programs with a fungicide treatment alone (i.e., prothioconazole + tebuconazole applied to a susceptible cultivar). The corresponding ranges for least squares mean test weight (Fig. 1F) were 709 to $732 \mathrm{~kg} / \mathrm{m}^{3}$ for programs with an MR cultivar, 736 to $752 \mathrm{~kg} / \mathrm{m}^{3}$ for those with an MS cultivar, and 684 to $714 \mathrm{~kg} / \mathrm{m}^{3}$ for those with a $\mathrm{S}$ cultivar. For both grain yield and test weight, the interquartile ranges were generally wider for programs with an MR or S cultivar than for those with an MS cultivar (Fig. 1E and F).

All management programs that included a tebuconazole + prothioconazole application resulted in significantly higher mean grain yield and test weight than the S_CK treatment, i.e., $\bar{D}$ was positive and significantly different from zero $(P<0.05$; Table 4 , Fig. $2 \mathrm{E})$. For grain yield, management programs that included an MS cultivar treated with tebuconazole + prothioconazole resulted in higher $\bar{D}$ values than programs in which an MR cultivar was used, or a fungicide treatment alone was applied. $\bar{D}$ for the former group of programs ranged from 541 to $753 \mathrm{~kg} / \mathrm{ha}$, with the highest values for the MS_A and MS_A+2 programs (Fig. 2E). The lowest overall mean yield increases relative to S_CK, -5 to $300 \mathrm{~kg} / \mathrm{ha}$, were observed for programs with an MR cultivar. Resistance alone (MR_CK) did not have a significantly higher mean grain yield than the $\mathrm{S} \_\mathrm{CK}$ treatment $(P=0.958$, Table 4).

Contrary to what was observed for grain yield, management programs with an MR cultivar treated with prothioconazole + tebuconazole had the highest $\bar{D}$ values for test weight, followed by programs with MS cultivars, and lastly by those in which a fungicide treatment alone was used (susceptible-treated). $\bar{D}$ ranged from 58.9 to $60.5 \mathrm{~kg} / \mathrm{m}^{3}$ for the former group of programs and from 23.6 to $25.3 \mathrm{~kg} / \mathrm{m}^{3}$ for the latter group (Table 4). Resistance alone (MR $\mathrm{CK})$ resulted in a higher mean test weight increase, relative to the S_CK $\left(45.6 \mathrm{~kg} / \mathrm{m}^{3}\right)$, than the fungicide treatment alone (Fig. 2F).

Influence of baseline FHB index on effect sizes. Mean FHB index for the S_CK treatment was used as a surrogate measure of conditions favorable for FHB development in the trials; trials with IND $_{\text {S_CK }}>10 \%$ were considered to be conducted under more

Table 2. Log response ratios (effect sizes) relative to a nontreated susceptible check and corresponding statistics from network meta-analysis of the effects of fungicide treatment $\times$ cultivar resistance class combinations on Fusarium head blight incidence (INC) and index (IND) in wheat

\begin{tabular}{|c|c|c|c|c|c|c|c|c|c|c|c|c|}
\hline \multirow[b]{2}{*}{ Management combination ${ }^{a}$} & \multicolumn{6}{|c|}{ INC $^{\mathbf{b}}$} & \multicolumn{6}{|c|}{ IND $^{\mathbf{b}}$} \\
\hline & $\bar{L}$ & SE & $\boldsymbol{P}$ & $C I_{L}$ & $C I_{U}$ & $\hat{\sigma}^{2}$ & $\bar{L}$ & SE & $\boldsymbol{P}$ & $C I_{L}$ & $C I_{U}$ & $\hat{\sigma}^{2}$ \\
\hline MR_A & -0.97 & 0.076 & $<0.001$ & -1.12 & -0.82 & 0.092 & -1.32 & 0.119 & $<0.001$ & -1.55 & -1.09 & 0.236 \\
\hline MR_A+2 & -1.17 & 0.084 & $<0.001$ & -1.33 & -1.00 & 0.058 & -1.44 & 0.136 & $<0.001$ & -1.71 & -1.17 & 0.268 \\
\hline MR_A+4 & -0.92 & 0.077 & $<0.001$ & -1.07 & -0.77 & 0.088 & -1.31 & 0.118 & $<0.001$ & -1.54 & -1.07 & 0.217 \\
\hline MR_A+6 & -0.91 & 0.078 & $<0.001$ & -1.06 & -0.75 & 0.086 & -1.24 & 0.111 & $<0.001$ & -1.46 & -1.02 & 0.149 \\
\hline MR_CK & -0.66 & 0.065 & $<0.001$ & -0.78 & -0.53 & 0.077 & -1.07 & 0.088 & $<0.001$ & -1.24 & -0.89 & 0.125 \\
\hline MS_A & -0.88 & 0.090 & $<0.001$ & -1.06 & -0.71 & 0.154 & -1.18 & 0.131 & $<0.001$ & -1.43 & -0.92 & 0.329 \\
\hline MS_A+2 & -0.96 & 0.087 & $<0.001$ & -1.13 & -0.79 & 0.108 & -1.26 & 0.127 & $<0.001$ & -1.51 & -1.01 & 0.248 \\
\hline MS_A+4 & -0.83 & 0.083 & $<0.001$ & -0.99 & -0.66 & 0.115 & -1.21 & 0.134 & $<0.001$ & -1.47 & -0.95 & 0.299 \\
\hline MS_A+6 & -0.83 & 0.086 & $<0.001$ & -1.00 & -0.66 & 0.116 & -1.13 & 0.129 & $<0.001$ & -1.38 & -0.87 & 0.248 \\
\hline MS_CK & -0.59 & 0.102 & $<0.001$ & -0.79 & -0.39 & 0.221 & -0.82 & 0.151 & $<0.001$ & -1.11 & -0.52 & 0.482 \\
\hline S_A & -0.31 & 0.054 & $<0.001$ & -0.42 & -0.21 & 0.057 & -0.51 & 0.069 & $<0.001$ & -0.64 & -0.37 & 0.090 \\
\hline S_A +2 & -0.42 & 0.076 & $<0.001$ & -0.57 & -0.27 & 0.112 & -0.60 & 0.081 & $<0.001$ & -0.76 & -0.44 & 0.103 \\
\hline S_A+4 & -0.30 & 0.056 & $<0.001$ & -0.41 & -0.19 & 0.056 & -0.52 & 0.075 & $<0.001$ & -0.67 & -0.38 & 0.094 \\
\hline S_A+6 & -0.33 & 0.053 & $<0.001$ & -0.43 & -0.22 & 0.045 & -0.48 & 0.074 & $<0.001$ & -0.63 & -0.34 & 0.095 \\
\hline
\end{tabular}

${ }^{a}$ Management combinations consisting of the application of the fungicide prothioconazole + tebuconazole at $50 \%$ early anthesis, or at 2,4 , or 6 days after $50 \%$ early anthesis $(\mathrm{A}, \mathrm{A}+2, \mathrm{~A}+4$, or $\mathrm{A}+6$, respectively) to moderately resistant (MR), moderately susceptible (MS), or susceptible (S) wheat cultivars.

${ }^{\mathrm{b}} \log$ response ratio $(\bar{L})$, standard error $(\mathrm{SE})$, level of significance $(P)$, lower and upper limits of the $95 \%$ confidence interval around $\bar{L}\left(C I_{L}\right.$ and $C I_{U}$, respectively), and between study variance $\left(\hat{\sigma}^{2}\right)$.

Table 3. Log response ratios (effect sizes) relative to a nontreated susceptible check and corresponding statistics from network meta-analysis of the effects of fungicide treatment $\times$ cultivar resistance class combinations on Fusarium damaged kernels (FDK) and deoxynivalenol (DON) in wheat

\begin{tabular}{|c|c|c|c|c|c|c|c|c|c|c|c|c|}
\hline \multirow[b]{2}{*}{ Management combination ${ }^{a}$} & \multicolumn{6}{|c|}{ FDK $^{\mathbf{b}}$} & \multicolumn{6}{|c|}{$\mathbf{D O N}^{\mathbf{b}}$} \\
\hline & $\bar{L}$ & SE & $P$ & $C I_{L}$ & $C I_{U}$ & $\hat{\sigma}^{2}$ & $\bar{L}$ & SE & $\boldsymbol{P}$ & $C I_{L}$ & $C I_{U}$ & $\hat{\sigma}^{2}$ \\
\hline MR_A & -1.23 & 0.162 & $<0.001$ & -1.55 & -0.91 & 0.365 & -0.93 & 0.093 & $<0.001$ & -1.11 & -0.75 & 0.193 \\
\hline MR_A+2 & -1.28 & 0.171 & $<0.001$ & -1.62 & -0.94 & 0.366 & -1.01 & 0.100 & $<0.001$ & -1.20 & -0.81 & 0.194 \\
\hline MR_A+4 & -1.07 & 0.134 & $<0.001$ & -1.33 & -0.81 & 0.219 & -1.03 & 0.099 & $<0.001$ & -1.22 & -0.83 & 0.214 \\
\hline MR_A+6 & -1.26 & 0.149 & $<0.001$ & -1.55 & -0.97 & 0.260 & -1.02 & 0.093 & $<0.001$ & -1.20 & -0.83 & 0.180 \\
\hline MR_CK & -1.09 & 0.158 & $<0.001$ & -1.40 & -0.78 & 0.370 & -0.81 & 0.076 & $<0.001$ & -0.96 & -0.65 & 0.127 \\
\hline MS_A & -1.15 & 0.163 & $<0.001$ & -1.47 & -0.83 & 0.395 & -0.86 & 0.109 & $<0.001$ & -1.08 & -0.65 & 0.258 \\
\hline MS_A+2 & -1.13 & 0.151 & $<0.001$ & -1.43 & -0.84 & 0.298 & -0.92 & 0.106 & $<0.001$ & -1.13 & -0.71 & 0.227 \\
\hline MS_A+4 & -1.09 & 0.155 & $<0.001$ & -1.39 & -0.78 & 0.352 & -0.89 & 0.111 & $<0.001$ & -1.11 & -0.67 & 0.261 \\
\hline MS_A+6 & -1.13 & 0.137 & $<0.001$ & -1.40 & -0.86 & 0.231 & -0.93 & 0.087 & $<0.001$ & -1.11 & -0.76 & 0.137 \\
\hline MS_CK & -0.84 & 0.167 & $<0.001$ & -1.17 & -0.51 & 0.450 & -0.61 & 0.094 & $<0.001$ & -0.80 & -0.43 & 0.193 \\
\hline S_A & -0.32 & 0.070 & $<0.001$ & -0.46 & -0.18 & 0.068 & -0.30 & 0.052 & $<0.001$ & -0.41 & -0.20 & 0.058 \\
\hline S_A+2 & -0.33 & 0.077 & $<0.001$ & -0.48 & -0.17 & 0.073 & -0.41 & 0.057 & $<0.001$ & -0.53 & -0.30 & 0.054 \\
\hline S_A+4 & -0.22 & 0.074 & 0.003 & -0.37 & -0.08 & 0.070 & -0.40 & 0.052 & $<0.001$ & -0.50 & -0.30 & 0.050 \\
\hline S_A+6 & -0.30 & 0.073 & $<0.001$ & -0.45 & -0.16 & 0.072 & -0.36 & 0.048 & $<0.001$ & -0.46 & -0.27 & 0.040 \\
\hline
\end{tabular}

${ }^{a}$ Management combinations consisting of the application of the fungicide prothioconazole + tebuconazole at $50 \%$ early anthesis, or at 2,4 , or 6 days after $50 \%$ early anthesis $(\mathrm{A}, \mathrm{A}+2, \mathrm{~A}+4$, or $\mathrm{A}+6$, respectively) to moderately resistant (MR), moderately susceptible (MS), or susceptible (S) wheat cultivars.

${ }^{\text {b }} \log$ response ratio $(\bar{L})$, standard error $(\mathrm{SE})$, level of significance $(P)$, lower and upper limits of the $95 \%$ confidence interval around $\bar{L}\left(C I_{L}\right.$ and $C I_{U}$, respectively), and between study variance $\left(\hat{\sigma}^{2}\right)$. 
favorable conditions for FHB development than those with IND $_{\mathrm{S}_{-} \mathrm{CK}}$ $\leq 10 \%$. Accounting for this trial-specific factor reduced the betweenstudy variability $\left(\sigma^{2}\right)$ of the $\bar{L}$ values for the effects of most management programs on four (test weight, FDK, IND, and DON) of the six measured responses. These reductions were generally higher for test weight than for FDK, IND, or DON. For IND (Tables 2 and 5), FDK (Tables 3 and 6), and DON (Tables 3 and 7), the reduction in $\sigma^{2}$ was generally higher for programs consisting of combinations of MR or
MS cultivars with a fungicide application than for fungicide application or resistance alone. For test weight, reductions in $\sigma^{2}$ were fairly consistent across management programs, ranging from 30 to $71 \%$.

Based on the results from the analyses with the moderator variable, there was a trend toward greater efficacy (lowest $\bar{L}$ and higher $\bar{C}$ values) against IND, DON, and FDK under favorable conditions (Tables 5,6 , and 7). For IND, the effect of IND $_{\mathrm{S}_{-} \mathrm{CK}}$ on $\bar{L}$ was statistically significant $(P<0.05$; Table 5$)$ for programs consisting of an MR or MS
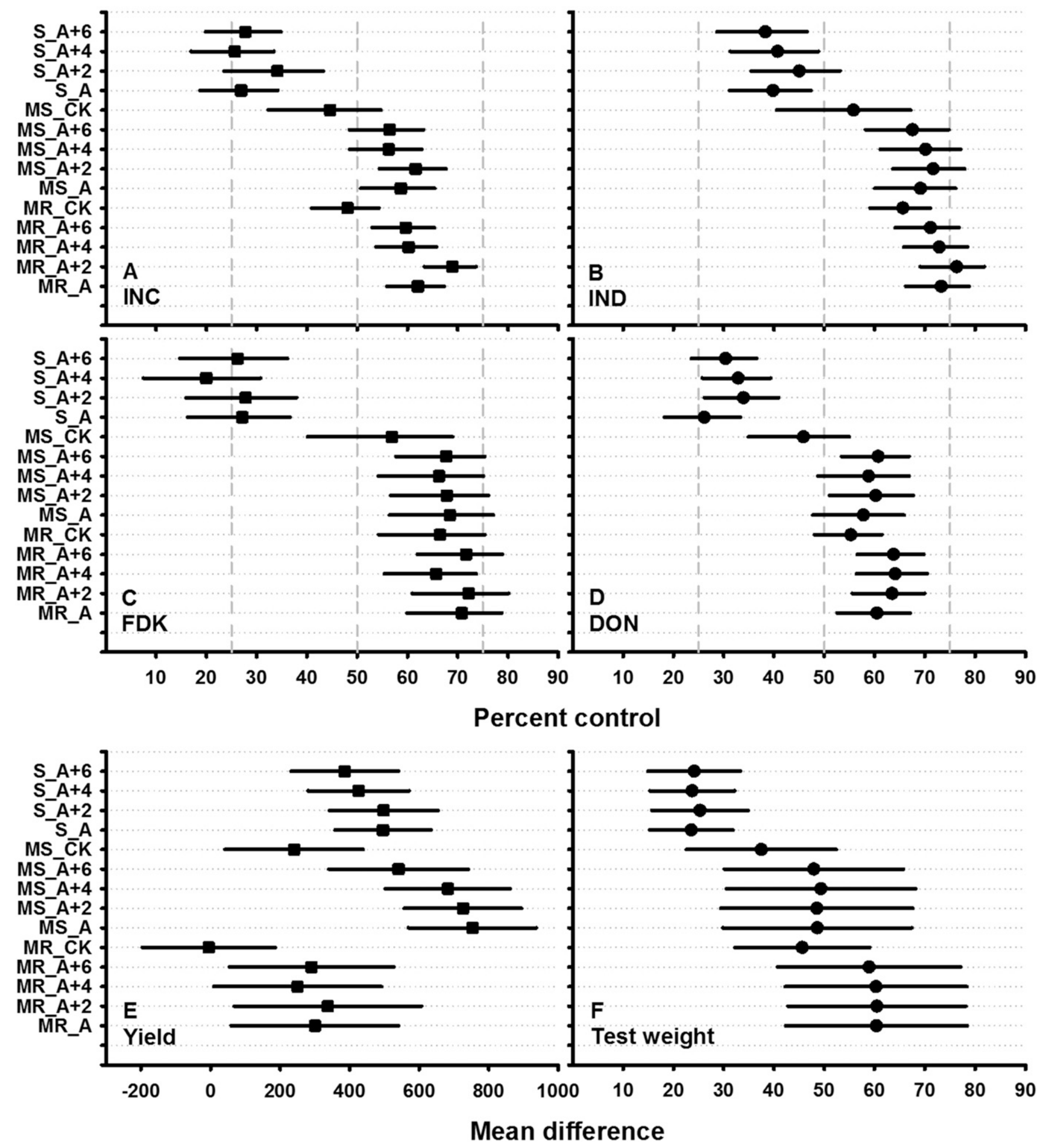

Fig. 2. Estimated overall mean percent control $(\bar{C})$ of Fusarium head blight incidence $(\mathbf{A})$ and index (B), Fusarium damaged kernels (C), deoxynivalenol (D), and mean difference $(\bar{D})$ in yield $(\mathbf{E})$ and test weight $(\mathbf{F})$ for different fungicide treatment by cultivar resistance class combinations relative to a nontreated susceptible check. Error bars are the $95 \%$ confidence intervals $(\mathrm{Cl})$ around $\bar{C}$ and $\bar{D}$. Percent controls and their Cls were estimated through back-transformation of log-response ratios and their $95 \% \mathrm{Cls}(\bar{C}=[1-\exp (\bar{L})] \cdot 100)$ from network meta-analyses. Management combinations consisted of the application of the fungicide prothioconazole + tebuconazole at $50 \%$ early anthesis, or at 2,4 , or 6 days after $50 \%$ early anthesis $(A, A+2, A+4$, or $A+6$, respectively) to moderately resistant (MR), moderately susceptible (MS), or susceptible (S) wheat cultivars. 
cultivar treated with prothioconazole + tebuconazole at $50 \%$ early anthesis or 2 to 5 days after early anthesis, indicating that relative to S_CK, the magnitude of the effect of these programs depended on the conditions under which the trials were conducted (Table 5). When MS or MR cultivars were treated at early anthesis or between 2 and 5 days after early anthesis (MR_A, MR_A+2, MR_A+4, MS_ A, and MS_A+2, MS_A+4), $\bar{C}$ values were 5 to $26 \%$ higher under FHB-favorable conditions. Efficacy was 4 to $10 \%$ greater for applications made at or close to $50 \%$ early anthesis compared with those made 6 or more days later under favorable conditions ( $\operatorname{IND}_{\mathrm{S} \text { CK }}>10 \%$ ), but the opposite was observed at IND $_{\text {S_CK }} \leq 10 \%$ (Table 5). On both MS and MR cultivars, the latest tested application timing (6 to 7 days after anthesis) was equally effective against IND at the two levels of the moderator variable (Table 5; $P>0.22$ for MS_A+6 and MR_A+6). The effect of fungicide application alone (S_A, S_ $\mathrm{A}+2, \mathrm{~S} \_\mathrm{A}+4$, and $\mathrm{S} \_\mathrm{A}+6$ ) on IND was not influenced by $\mathrm{IND}_{\mathrm{S} \_\mathrm{CK}}$ (Table 5; $P>0.11$ ).
Similar trends to those observed for IND were observed for FDK, but for the latter response, IND $_{\mathrm{S} \_\mathrm{CK}}$ did not only affect the magnitude but also the significance of the effect sizes. The effect of IND $_{\mathrm{S}_{-} \mathrm{CK}}$ on $\bar{L}$ was statistically significant (Table $6 ; P<0.05$ ) for all but three of the management programs with MR cultivars (MR_CK, MR_A+4, and MR_A+6) and three of the programs with $S$ cultivars (S_A+2, S_A+4, and S_A+6), and in all cases, $\bar{C}$ values were considerably higher under favorable (IND S $C K_{C}>10 \%$ ) compared with less favorable $\left(\mathrm{IND}_{\mathrm{S} \_\mathrm{CK}} \leq 10 \%\right)$ conditions for FHB development. When MR cultivars were treated with the fungicide at $50 \%$ early anthesis or 2 to 3 days later, the difference in $\bar{C}$ values between the two categories of the moderator variable was $22 \%$ (Table 6). For programs with an MS cultivar, $\bar{C}$ values were 21 to $45 \%$ higher under more favorable conditions for FHB than under less favorable conditions. The effects of management programs that included a susceptible cultivar were generally not statistically significant when conditions were relatively less favorable for FHB $(P>0.05$; Table 6). The only

Table 4. Estimated mean differences in grain yield $(\mathrm{kg} / \mathrm{ha})$ and test weight $\left(\mathrm{kg} / \mathrm{m}^{3}\right)$ relative to a nontreated susceptible check and corresponding statistics from network meta-analyses of the effects of fungicide treatment $\times$ cultivar resistance class combinations on Fusarium head blight in wheat

\begin{tabular}{|c|c|c|c|c|c|c|c|c|c|c|c|c|}
\hline \multirow[b]{2}{*}{ Management combination ${ }^{a}$} & \multicolumn{6}{|c|}{ Grain yield $^{\mathbf{b}}$} & \multicolumn{6}{|c|}{ Test weight $^{\text {b }}$} \\
\hline & $\bar{D}$ & SE & $\boldsymbol{P}$ & $C \boldsymbol{I}_{L}$ & $C \boldsymbol{I}_{U}$ & $\hat{\sigma}^{2}$ & $\bar{D}$ & SE & $\boldsymbol{P}$ & $C I_{L}$ & $\boldsymbol{C I}_{U}$ & $\hat{\sigma}^{2}$ \\
\hline MR_A & 300.10 & 122.25 & 0.015 & 59.59 & 540.61 & 318.30 & 60.39 & 9.172 & $<0.001$ & 42.34 & 78.44 & 2.10 \\
\hline MR_A+2 & 337.12 & 136.89 & 0.014 & 67.82 & 606.41 & 373.80 & 60.49 & 9.004 & $<0.001$ & 42.77 & 78.21 & 1.96 \\
\hline MR_A+4 & 250.06 & 122.67 & 0.042 & 8.75 & 491.38 & 311.23 & 60.31 & 9.182 & $<0.001$ & 42.24 & 78.37 & 2.09 \\
\hline MR_A+6 & 290.65 & 120.11 & 0.016 & 54.36 & 526.95 & 284.59 & 58.94 & 9.257 & $<0.001$ & 40.72 & 77.15 & 2.12 \\
\hline MR_CK & -5.11 & 97.29 & 0.958 & -196.51 & 186.29 & 170.45 & 45.64 & 6.821 & $<0.001$ & 32.22 & 59.06 & 1.11 \\
\hline MS_A & 752.87 & 93.59 & $<0.001$ & 568.75 & 937.00 & 139.58 & 48.64 & 9.555 & $<0.001$ & 29.84 & 67.44 & 2.28 \\
\hline MS_A+2 & 725.69 & 85.96 & $<0.001$ & 556.58 & 894.80 & 73.46 & 48.54 & 9.708 & $<0.001$ & 29.43 & 67.64 & 2.33 \\
\hline MS_A+4 & 682.57 & 91.32 & $<0.001$ & 502.92 & 862.23 & 114.95 & 49.35 & 9.543 & $<0.001$ & 30.57 & 68.13 & 2.27 \\
\hline MS_A+6 & 540.51 & 102.03 & $<0.001$ & 339.79 & 741.23 & 152.39 & 47.95 & 9.059 & $<0.001$ & 30.13 & 65.78 & 2.01 \\
\hline MS_CK & 239.85 & 100.98 & 0.018 & 41.19 & 438.51 & 175.68 & 37.49 & 7.571 & $<0.001$ & 22.59 & 52.39 & 1.40 \\
\hline S_A & 495.99 & 70.08 & $<0.001$ & 358.13 & 633.86 & 50.25 & 23.57 & 4.219 & $<0.001$ & 15.27 & 31.87 & 0.38 \\
\hline S_A+2 & 497.91 & 79.40 & $<0.001$ & 341.72 & 654.11 & 47.47 & 25.31 & 4.885 & $<0.001$ & 15.70 & 34.93 & 0.48 \\
\hline S_A+4 & 425.81 & 73.90 & $<0.001$ & 280.42 & 571.20 & 51.38 & 23.75 & 4.289 & $<0.001$ & 15.31 & 32.19 & 0.37 \\
\hline S_A+6 & 385.92 & 78.49 & $<0.001$ & 231.50 & 540.34 & 66.73 & 24.14 & 4.675 & $<0.001$ & 14.94 & 33.34 & 0.46 \\
\hline
\end{tabular}

${ }^{a}$ Management combinations consisting of the application of the fungicide prothioconazole + tebuconazole at $50 \%$ early anthesis, or at 2 , 4 , or 6 days after $50 \%$ early anthesis (A, $\mathrm{A}+2, \mathrm{~A}+4$, or $\mathrm{A}+6$, respectively) to moderately resistant (MR), moderately susceptible (MS), or susceptible (S) wheat cultivars.

${ }^{\mathrm{b}} \bar{D}=$ mean difference between each management combination and the nontreated susceptible check, $\mathrm{SE}=$ standard error of $\bar{D}, P=$ significance level, $C I_{U}$ and $C I_{L}=$ upper and lower limits of the $95 \%$ confidence interval around $\bar{D}$, respectively, and $\hat{\sigma}^{2}=$ between study variance $(\times 1,000)$

Table 5. Overall mean log response ratios (effect sizes), percent control, and corresponding statistics for the influence of baseline Fusarium head blight index (IND) on the effects of fungicide treatment $\times$ cultivar resistance class combinations on Fusarium head blight index in wheat

\begin{tabular}{|c|c|c|c|c|c|c|c|c|c|c|c|c|c|c|c|c|}
\hline \multirow[b]{2}{*}{ Mgmt combo $^{\mathbf{a}}$} & \multicolumn{7}{|c|}{ High baseline $\mathbf{I N D}^{\mathbf{b}}$} & \multicolumn{7}{|c|}{ Low baseline IND ${ }^{b}$} & \multirow[b]{2}{*}{$P^{c}$} & \multirow[b]{2}{*}{$\hat{\sigma}^{2} \mathbf{d}$} \\
\hline & $\bar{L}$ & SE & $C I_{L}$ & $C I_{U}$ & $\overline{\boldsymbol{C}}$ & $C I_{L}$ & $C \boldsymbol{I}_{U}$ & $\bar{L}$ & SE & $C I_{L}$ & $C \boldsymbol{I}_{U}$ & $\overline{\boldsymbol{C}}$ & $C I_{L}$ & $C I_{U}$ & & \\
\hline MR_A & $-1.58^{*}$ & 0.183 & -1.94 & .22 & 79.30 & .33 & 85.56 & $-1.00 *$ & 0.148 & -1.29 & -0.70 & .06 & 50.57 & 72.40 & 0.014 & 0.18 \\
\hline MR_A+2 & $1.68^{*}$ & 0.200 & -2.08 & -1.29 & 81.39 & 72.42 & 87.45 & $-1.08 *$ & 0.163 & -1.40 & -0.76 & .95 & 53.05 & 5.31 & .020 & 0.17 \\
\hline MR_A+4 & $1.49 *$ & 0.169 & -1.83 & -1.16 & 77.56 & 68.73 & 83.90 & $-1.02 *$ & 0.155 & -1.32 & -0.71 & 63.87 & 51.00 & 73.36 & 0.038 & 0.18 \\
\hline MR_A+6 & $1.34 *$ & 0.164 & -1.66 & -1.02 & 73.87 & 63.95 & 81.06 & $-1.07 *$ & 0.157 & -1.38 & -0.76 & 65.64 & 53.24 & 74.74 & 0.227 & 0.16 \\
\hline MR_CK & $1.19^{*}$ & 0.122 & -1.43 & -0.94 & 69.43 & 61.12 & 75.96 & $-0.90^{*}$ & 0.121 & -1.14 & -0.66 & 59.44 & 48.50 & 68.06 & 0.102 & 0.11 \\
\hline MS_A & $-1.48^{*}$ & 0.163 & -1.80 & -1.16 & 77.26 & 68.64 & 83.51 & $-0.74 *$ & 0.164 & -1.06 & -0.41 & 52.08 & 33.80 & 65.31 & 0.001 & 0.20 \\
\hline MS_A+ & $55 \%$ & 0.159 & -1.86 & -1.23 & 78.69 & 70.85 & 84.42 & $-0.85^{*}$ & 0.162 & -1.17 & -0.54 & 57.46 & 41.49 & 69.06 & 0.003 & 0.14 \\
\hline MS_A+4 & $.45^{*}$ & 0.165 & -1.77 & -1.12 & 76.51 & 67.49 & 83.03 & $-0.87 *$ & 0.190 & -1.24 & -0.49 & 57.92 & 38.80 & 71.07 & 0.021 & 0.22 \\
\hline MS_A+6 & $1.17 *$ & 0.156 & -1.47 & -0.86 & 68.89 & 57.74 & 77.09 & $-1.02 *$ & 0.195 & -1.40 & -0.64 & 63.92 & 47.06 & 75.41 & 0.553 & 0.21 \\
\hline $\mathrm{CK}$ & 1 & 0.186 & -1.47 & -0.74 & 66.88 & 52.20 & 77.05 & $-0.40^{\mathrm{n}}$ & 0.221 & -0 & 0.04 & 32.70 & -3.97 & 56.44 & 0.015 & 0.40 \\
\hline $\mathrm{S} \_$ & $60 *$ & 0.098 & -0.79 & -0.41 & 45.04 & 33.34 & 54.69 & $-0.37 *$ & 0.104 & -0.57 & -0.17 & 30.99 & 15.40 & 43.71 & 0.112 & 0.10 \\
\hline S_A+2 & $.57 *$ & 0.108 & -0.78 & -0.36 & 43.40 & 30.02 & 54.22 & $-0.64 *$ & 0.129 & -0.89 & -0.39 & 47.26 & 32.02 & 59.08 & 0.675 & 0.11 \\
\hline S_A+4 & $0.50^{*}$ & 0.096 & -0.68 & -0.31 & 39.05 & 26.45 & 49.49 & $-0.55^{*}$ & 0.122 & -0.79 & -0.31 & 42.47 & 26.84 & 54.76 & 0.710 & 0.09 \\
\hline S_A+6 & $-0.41^{*}$ & 0.093 & -0.59 & -0.22 & 33.51 & 20.12 & 44.66 & $-0.56^{*}$ & 0.113 & -0.78 & -0.34 & 42.92 & 28.70 & 54.30 & 0.299 & 0.09 \\
\hline
\end{tabular}

a Management combinations consisting of the application of the fungicide prothioconazole + tebuconazole at 50\% early anthesis, or at 2, 4, or 6 days after $50 \%$ early anthesis $(\mathrm{A}, \mathrm{A}+2, \mathrm{~A}+4$, or $\mathrm{A}+6$, respectively) to moderately resistant (MR), moderately susceptible (MS), or susceptible (S) wheat cultivars.

${ }^{\mathrm{b}}$ High and low baseline IND, used here as surrogates for more and less favorable conditions for FHB development, were represented by mean FHB index in the nontreated susceptible check $>10 \%$ and $\leq 10 \%$, respectively; $\bar{L}=$ mean log of the response ratio relative to a nontreated check [* denotes the statistical significance of $\bar{L}(P<0.05)$, whereas n denotes not statistically significant], SE = standard error of $\bar{L}, C I_{L}$ and $C I_{U}=$ lower and upper limits of the $95 \%$ confidence interval around $\bar{L}$, respectively, $\bar{C}=$ mean percent control estimated as $\bar{C}=[1-\exp (\bar{L})] \cdot 100 ; C I_{L}$ and $C I_{U}=$ lower and upper limits of the $95 \%$ confidence interval around $\bar{C}$, respectively.

${ }^{\mathrm{c}} P=$ significance level for the effect of baseline FHB index.

$\mathrm{d} \hat{\sigma}^{2}=$ between study variance. 
exception was for $\mathrm{S} \_A+6$. Depending on the resistance reaction of the cultivar treated, earlier applications reduced FDK by up to $11 \%$ under conditions favorable for FHB compared with later applications (Table 6).

For DON, the effect of IND $_{\text {S CK }}$ on $\bar{L}$ was statistically significant $(P<0.05$; Table 7) for all management programs that include a prothioconazole + tebuconazole application except for those consisting of a late application to a susceptible cultivar (S_A+2, S_A+4, and S_ $\mathrm{A}+6)$ or an application to an MS cultivar at 6 to 7 days after anthesis (MS_A+6). Under favorable conditions ( IND $_{\mathrm{S} \_\mathrm{CK}}>10 \%$ ), $\bar{C}$ values were 16 to $18 \%$ higher for management programs including an MR cultivar and 9 to $24 \%$ higher for programs with an MS cultivar, when compared with the efficacy of the same programs under less FHBconducive conditions $\left(\mathrm{IND}_{\mathrm{S}_{\mathrm{C}} \mathrm{CK}} \leq 10 \%\right)$. At both levels of $\mathrm{IND}_{\mathrm{S}}$ $\mathrm{CK}$, efficacy against DON was comparable between anthesis (50\% early anthesis) and post-anthesis (after 50\% early anthesis) applications or greater for post-anthesis applications, particularly on susceptible cultivars (Table 7).

The effect of $\mathrm{IND}_{\mathrm{S}_{-} \mathrm{CK}}$ on yield response $(\bar{D})$ was statistically significant $(P<0.05)$ for only two of the 14 tested management programs, S_A and MS_A (prothioconazole + tebuconazole applied to a susceptible or a moderately susceptible cultivar, respectively, at anthesis). $\bar{D}$ was 928 (SE 113$) \mathrm{kg} / \mathrm{ha}$ at $\mathrm{IND}_{\mathrm{S} \_\mathrm{CK}}>10 \%$ and 439 $(\mathrm{SE} 139) \mathrm{kg} / \mathrm{ha}$ at $\mathrm{IND}_{\mathrm{S} \_\mathrm{CK}} \leq 10 \%$ for MS_A, and 650 (SE 100)

Table 6. Overall mean log response ratios (effect sizes), percent control, and corresponding statistics for the influence of baseline Fusarium head blight index (IND) on the effects of fungicide treatment $\times$ cultivar resistance class combinations on Fusarium damaged kernels in wheat

\begin{tabular}{|c|c|c|c|c|c|c|c|c|c|c|c|c|c|c|c|c|}
\hline \multirow[b]{2}{*}{ Mgmt combo $^{\mathbf{a}}$} & \multicolumn{7}{|c|}{ High baseline IND $^{\mathbf{b}}$} & \multicolumn{7}{|c|}{ Low baseline IND ${ }^{b}$} & \multirow[b]{2}{*}{$P^{\mathbf{c}}$} & \multirow[b]{2}{*}{$\hat{\sigma}^{2} \mathbf{d}$} \\
\hline & $\bar{L}$ & SE & $C I_{L}$ & $C I_{U}$ & $\overline{\boldsymbol{C}}$ & $C I_{L}$ & $C I_{U}$ & $\bar{L}$ & SE & $C I_{L}$ & $C I_{U}$ & $\bar{C}$ & $C I_{L}$ & $C I_{U}$ & & \\
\hline MR_A & $-1.42 *$ & 0.186 & -1.79 & -1.06 & 75.94 & 65.31 & 83.32 & $-0.77 *$ & 0.261 & -1.28 & -0.25 & 53.48 & 22.15 & 72.20 & .041 & 0.30 \\
\hline MR_A+2 & $-1.48^{*}$ & 0.200 & -1.88 & -1.09 & 77.33 & 66.36 & 84.72 & $-0.81^{*}$ & 0.272 & -1.35 & -0.27 & 55.50 & 23.94 & 73.96 & .047 & 0.30 \\
\hline MR_A+4 & $-1.16^{*}$ & 0.192 & -1.54 & -0.78 & 68.78 & 54.38 & 78.63 & $-1.03^{*}$ & 0.281 & -1.58 & -0.47 & 64.16 & 37.65 & 79.39 & 0.686 & 0.36 \\
\hline MR_A+6 & $-1.38 *$ & 0.175 & -1.72 & -1.03 & 74.81 & 64.41 & 82.17 & $-0.95^{*}$ & 0.246 & -1.44 & -0.47 & 61.41 & 37.38 & 76.21 & 0.159 & 0.23 \\
\hline MR_CK & $-1.21 *$ & 0.190 & -1.59 & -0.84 & 70.26 & 56.73 & 79.56 & $-0.75^{*}$ & 0.282 & -1.31 & -0.20 & 52.85 & 17.75 & 72.97 & 0.177 & 0.37 \\
\hline MS_A & $-1.47 *$ & 0.167 & -1.80 & -1.14 & 77.04 & 68.12 & 83.47 & $-0.52^{*}$ & 0.218 & -0.95 & -0.09 & 40.38 & 8.37 & 61.20 & 0.001 & 0.21 \\
\hline MS_A+2 & $-1.36^{*}$ & 0.156 & -1.67 & -1.05 & 74.28 & 65.04 & 81.08 & $-0.61^{*}$ & 0.231 & -1.07 & -0.15 & 45.68 & 14.29 & 65.57 & 0.008 & 0.19 \\
\hline MS_A+4 & $-1.31 *$ & 0.155 & -1.61 & -1.00 & 72.88 & 63.19 & 80.02 & $-0.53 *$ & 0.223 & -0.97 & -0.09 & 41.15 & 8.66 & 62.09 & 0.005 & 0.20 \\
\hline MS_A+6 & $-1.30 *$ & 0.146 & -1.59 & -1.01 & 72.80 & 63.72 & 79.61 & $-0.72^{*}$ & 0.209 & -1.13 & -0.31 & 51.47 & 26.78 & 67.84 & 0.024 & 0.15 \\
\hline MS_CK & $.09 *$ & 0.166 & -1.41 & -0.76 & 66.26 & 53.18 & 75.69 & $-0.24^{\mathrm{n}}$ & 0.245 & -0.73 & 0.24 & 21.66 & -27.02 & 51.68 & 0.005 & 0.28 \\
\hline S_f & $42 *$ & 0.081 & -0.58 & -0.26 & 34.39 & 23.00 & 44.09 & $-0.09^{n}$ & 0.116 & -0.32 & 0.14 & 8.34 & -15.18 & 27.06 & 0.019 & 0.06 \\
\hline S_A+2 & $-0.37 *$ & 0.084 & -0.54 & -0.21 & 31.19 & 18.74 & 41.74 & $-0.19^{\mathrm{n}}$ & 0.130 & -0.45 & 0.06 & 17.69 & -6.39 & 36.32 & 0.250 & 0.06 \\
\hline S_A+4 & $-0.27^{*}$ & 0.077 & -0.42 & -0.11 & 23.40 & 10.76 & 34.24 & $-0.12^{\mathrm{n}}$ & 0.130 & -0.38 & 0.14 & 11.26 & -14.69 & 31.34 & 0.333 & 0.05 \\
\hline S_A+6 & $-0.29 *$ & 0.083 & -0.45 & -0.12 & 24.87 & 11.45 & 36.25 & $-0.38^{*}$ & 0.131 & -0.64 & -0.12 & 31.62 & 11.40 & 47.22 & 0.546 & 0.07 \\
\hline
\end{tabular}

${ }^{a}$ Management combinations consisting of the application of the fungicide prothioconazole + tebuconazole at $50 \%$ early anthesis, or at 2,4 , or 6 days after $50 \%$ early anthesis $(\mathrm{A}, \mathrm{A}+2, \mathrm{~A}+4$, or $\mathrm{A}+6$, respectively) to moderately resistant (MR), moderately susceptible (MS), or susceptible (S) wheat cultivars.

${ }^{\mathrm{b}}$ High and low baseline IND, used here as surrogates for more and less favorable conditions for FHB development, were represented by mean FHB index in the nontreated susceptible check $>10 \%$ and $\leq 10 \%$, respectively; $\bar{L}=$ mean log of the response ratio relative to a nontreated check [* denotes the statistical significance of $\bar{L}(P<0.05)$, whereas n denotes not statistically significant], SE = standard error of $\bar{L}, C I_{L}$ and $C I_{U}=1$ lower and upper limits of the $95 \%$ confidence interval around $\bar{L}$, respectively, $\bar{C}=$ mean percent control estimated as $\bar{C}=[1-\exp (\bar{L})] \cdot 100 ; C I_{L}$ and $C I_{U}=$ lower and upper limits of the $95 \%$ confidence interval around $\bar{C}$, respectively.

c $P=$ significance level for the effect of baseline FHB index.

d $\hat{\sigma}^{2}=$ between study variance.

Table 7. Overall mean log response ratio (effect sizes), percent control, and corresponding statistics for the influence of baseline Fusarium head blight index (IND) level on the effects of fungicide treatment $\times$ cultivar resistance class combinations on deoxynivalenol (DON) contamination of wheat grain

\begin{tabular}{|c|c|c|c|c|c|c|c|c|c|c|c|c|c|c|c|c|}
\hline \multirow[b]{2}{*}{ Mgmt comboa $^{a}$} & \multicolumn{7}{|c|}{ High baseline IND ${ }^{b}$} & \multicolumn{7}{|c|}{ Low baseline IND ${ }^{b}$} & \multirow[b]{2}{*}{$P^{c}$} & \multirow[b]{2}{*}{$\hat{\sigma}^{2 \mathbf{d}}$} \\
\hline & $\bar{L}$ & SE & $C I_{L}$ & $C I_{U}$ & $\bar{C}$ & $C I_{L}$ & $C I_{U}$ & $\bar{L}$ & SE & $C I_{L}$ & $C I_{U}$ & $\bar{C}$ & $C I_{L}$ & $C I_{U}$ & & \\
\hline MR_A & $-1.11 *$ & 0.111 & -1.33 & -0.90 & 67.19 & 59.18 & 73.63 & $-0.68 *$ & 0.126 & -0.92 & -0.43 & 49.09 & 34.79 & 60.25 & 0.009 & 0.15 \\
\hline MR_A+2 & $-1.20 *$ & 0.116 & -1.43 & -0.97 & 69.86 & 62.13 & 76.02 & $-0.75 *$ & 0.136 & -1.02 & -0.48 & 52.70 & 38.18 & 63.82 & 0.012 & 0.14 \\
\hline MR_A+4 & $-1.20 *$ & 0.118 & -1.44 & -0.97 & 69.99 & 62.15 & 76.21 & $-0.77 *$ & 0.135 & -1.04 & -0.51 & 53.92 & 39.90 & 64.67 & 0.017 & 0.16 \\
\hline MR_A+6 & $-1.20 *$ & 0.108 & -1.41 & -0.99 & 69.85 & 62.68 & 75.64 & $-0.76^{*}$ & 0.123 & -1.00 & -0.52 & 53.20 & 40.38 & 63.26 & 0.008 & 0.13 \\
\hline MR_CK & $-0.91 *$ & 0.096 & -1.10 & -0.72 & 59.70 & 51.36 & 66.62 & $-0.66 *$ & 0.110 & -0.87 & -0.44 & 48.11 & 35.55 & 58.21 & 0.084 & 0.11 \\
\hline MS_A & $-1.11 *$ & 0.128 & -1.36 & -0.86 & 67.13 & 57.72 & 74.45 & $-0.56^{*}$ & 0.151 & -0.86 & -0.27 & 43.04 & 23.39 & 57.65 & 0.006 & 0.20 \\
\hline MS_A+2 & $-1.11^{*}$ & 0.126 & -1.36 & -0.86 & 66.98 & 57.66 & 74.25 & $-0.68 *$ & 0.156 & -0.98 & -0.37 & 49.13 & 30.78 & 62.61 & 0.032 & 0.18 \\
\hline MS_A+4 & $-1.08 *$ & 0.131 & -1.34 & -0.82 & 66.09 & 56.10 & 73.81 & $-0.63 *$ & 0.164 & -0.95 & -0.31 & 46.83 & 26.58 & 61.50 & 0.033 & 0.21 \\
\hline MS_A+6 & $-1.03 *$ & 0.106 & -1.24 & -0.83 & 64.40 & 56.18 & 71.08 & $-0.80 *$ & 0.136 & -1.07 & -0.54 & 55.26 & 41.51 & 65.78 & 0.186 & 0.12 \\
\hline MS_CK & $-0.80 *$ & 0.110 & -1.02 & -0.58 & 55.00 & 44.12 & 63.76 & $-0.36 *$ & 0.132 & -0.63 & -0.10 & 30.57 & 9.92 & 46.49 & 0.012 & 0.15 \\
\hline S_A & $-0.41 *$ & 0.067 & -0.54 & -0.28 & 33.53 & 24.23 & 41.69 & $-0.17 *$ & 0.076 & -0.32 & -0.02 & 15.75 & 2.20 & 27.43 & 0.019 & 0.05 \\
\hline S_A+2 & $-0.46^{*}$ & 0.072 & -0.60 & -0.32 & 36.71 & 27.02 & 45.11 & $-0.36 *$ & 0.090 & -0.53 & -0.18 & 30.05 & 16.47 & 41.43 & 0.388 & 0.05 \\
\hline S_A+4 & $-0.46^{*}$ & 0.064 & -0.59 & -0.33 & 36.87 & 28.38 & 44.35 & $-0.32 *$ & 0.080 & -0.47 & -0.16 & 27.25 & 14.92 & 37.79 & 0.166 & 0.05 \\
\hline S_A+6 & $-0.39 *$ & 0.061 & -0.51 & -0.27 & 32.29 & 23.60 & 40.00 & $-0.33 *$ & 0.073 & -0.47 & -0.18 & 27.96 & 16.81 & 37.61 & 0.516 & 0.04 \\
\hline
\end{tabular}

a Management combinations consisting of the application of the fungicide prothioconazole + tebuconazole at $50 \%$ early anthesis, or at 2,4 , or 6 days after $50 \%$ early anthesis $(\mathrm{A}, \mathrm{A}+2, \mathrm{~A}+4$, or $\mathrm{A}+6$, respectively) to moderately resistant (MR), moderately susceptible (MS), or susceptible (S) wheat cultivars.

${ }^{\mathrm{b}}$ High and low baseline IND, used here as surrogates for more and less favorable conditions for FHB development, were represented by mean FHB index in the nontreated susceptible check $>10 \%$ and $\leq 10 \%$, respectively; $\bar{L}=$ mean log of the response ratio relative to a nontreated check [* denotes the statistical significance of $\bar{L}(P<0.05)], \mathrm{SE}=$ standard error of $\bar{L}, C I_{L}$ and $C I_{U}=$ lower and upper limits of the $95 \%$ confidence interval around $\bar{L}$, respectively, $\bar{C}=$ mean percent control estimated as $\bar{C}=[1-\exp (\bar{L})] \cdot 100 ; C I_{L}$ and $C I_{U}=$ lower and upper limits of the $95 \%$ confidence interval around $\bar{C}$, respectively

${ }^{\mathrm{c}} P=$ significance level for the effect of baseline FHB index.

d $\hat{\sigma}^{2}=$ between study variance. 


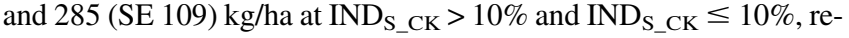
spectively, for $\mathrm{S}_{-} \mathrm{A}$. On the other hand, IND $\mathrm{S}_{\mathrm{CK}}$ had a highly significant effect $(P<0.001)$ on the test weight response to all management programs tested. $\bar{D}$ values were positive and significantly different from zero at $\mathrm{IND}_{\mathrm{S}_{-} \mathrm{CK}}>10 \%$, but not at $\mathrm{IND}_{\mathrm{S}_{\mathrm{CCK}}} \leq 10 \%$. In other words, the magnitude and significance of the effects of management programs on test weight were greater under conditions favorable for FHB than under less favorable conditions. At $\mathrm{IND}_{\mathrm{S} \_\mathrm{CK}}>10 \%, \bar{D}$ for MR_A, MR_A+2, MR_A+4, MR_A+6, and MR_CK were 85 (SE 8.3), 86 (SE 8.0), 85 (SE 8.7), 84 (SE 8.2), and 64 (SE 6.2) kg/m respectively. The corresponding values for management programs with an MS cultivar were 75 (SE 7.5), 76 (SE 7.6), 75 (SE 7.9), 70 (SE 9.0), and 59 (SE 5.7) $\mathrm{kg} / \mathrm{m}^{3}$, compared with 33 (SE 4.2), 38 (SE 4.6), 32 (SE 4.5), and 33 (SE 5.4) $\mathrm{kg} / \mathrm{m}^{3}$ for the S_A, S_A+2, S_A+4, and S_A+6 programs, respectively.

Predicted efficacy against IND, FDK, and DON in a future trial. Figure 3 shows estimated probabilities of achieving 50 and $75 \%$ control of IND ( $p_{50}$ and $p_{75}$, respectively), FDK, and DON in a future trial with each management program under more and less favorable conditions for FHB $\left(\right.$ IND $_{\mathrm{S}_{\mathrm{CK}}}>10 \%$ and $I_{\text {IND_CK }} \leq 10 \%$, respectively). For all three responses, by definition, $p_{50}$ values for a given management program were larger than corresponding $p_{75}$ values, and both were higher at $\mathrm{IND}_{\mathrm{S}_{-} \mathrm{CK}}>10 \%$ than at $\mathrm{IND}_{\mathrm{S}_{-} \mathrm{CK}} \leq 10 \%$. For instance, with an estimated $\bar{L}$ value of -0.99 and $\hat{\sigma}^{2}$ of $0.18(\hat{\sigma}=\sqrt{0.18}=0.42)($ Table 5$), p_{50}$ was estimated as $\varnothing((-0.69+0.99) / 0.42)=0.76$ and $p_{75}$ as $\varnothing((-1.39+0.99) / 0.42)=$ 0.18 for MR_A at low baseline levels of FHB (Fig. 3A).

At both high and low baseline levels of IND, management programs consisting of an application of prothioconazole + tebuconazole to an MR or MS cultivar had higher $p_{50}$ and $p_{75}$ values for IND than for a fungicide application alone (Fig. 3A and B). In fact, at both levels of the moderator variable, the chance of achieving a $75 \%$ control of IND with a fungicide treatment alone (S_A, S_A+ $2, \mathrm{~S} \_A+4$, or S_A+6) was close to zero, and the chance of a $50 \%$ control was less than $45 \%$ (Fig. 3A and B). The probabilities of seeing a 50 or $75 \%$ control of IND under FHB favorable conditions (Fig. 3B) were considerably higher with resistance alone (MR_CK or MS_CK) than with prothioconazole + tebuconazole alone (on susceptible cultivars). At $\mathrm{IND}_{\mathrm{S}_{\mathrm{CK}}} \leq 10 \%$, probability values were numerically higher for programs with MR or MS cultivars when applications were made after than at $50 \%$ early anthesis (Fig. 3A). On the other hand, at $\mathrm{IND}_{\mathrm{S} \_\mathrm{CK}}>10 \%, p_{50}$ values were more consistent (all above 0.85 ) across application timings for the MS and MR cultivars, whereas $p_{75}$ values decreased when the application was delayed by more than 3 days after $50 \%$ early anthesis, for a given cultivar resistance class.

Comparable results to those observed for IND were observed for FDK and DON (Fig. 3C, D, E, and F), with management programs consisting of an MR or MS cultivar treated with prothioconazole + tebuconazole having higher $p_{50}$ and $p_{75}$ values than fungicide-only programs (prothioconazole + tebuconazole applied to a susceptible cultivar) for both grain quality traits. However, for any given management program, probability values for FDK and DON were lower than the corresponding values for IND, particularly at low baseline levels of disease (Fig. 3A, C, and E). The chance of achieving a $75 \%$ control of FDK or DON under less favorable conditions for FHB were low (below 30\%) for some management programs and extremely low (below 5\%) for others (Fig. 3C and E). Under conditions favorable for FHB, the use of resistance alone (MR_CK or MS_CK) was considerably less likely to result in a 50 or $75 \%$ control of FDK or DON relative to S_CK than the use of an integrated approach (Fig. $3 \mathrm{D}$ and $\mathrm{F})$. Comparing application timings, treatments applied after $50 \%$ early anthesis had higher $p_{50}$ values for DON than those made at $50 \%$ early anthesis (Fig. 3E and F).

Economic benefit of management programs. Using Equations 3 and 4, economic benefit was estimated as the difference in net cash income (NCI) between each management program and the reference program (S_CK). For the purpose of this analysis, summary statistics from the distributions of least squares mean grain yield, test weight,
FDK, and DON for the nontreated susceptible check (S_CK) in Figure 1 were used as baseline (starting) responses to estimate $N C I$ for S_CK $\left(\mathrm{NCI}_{S_{-} \mathrm{CK}}\right)$. First we obtained estimates of the overall means for yield ( $Y l d ; 4.3 \mathrm{MT} / \mathrm{ha})$ and test weight $\left(684 \mathrm{~kg} / \mathrm{m}^{3}\right)$ for $\mathrm{S}_{-}$ CK from Figure $1 \mathrm{E}$ and $\mathrm{F}$, and the first and third quartiles of the distributions of FDK (10.5 and 27.6\%, respectively) and DON (2.3 and $8.2 \mathrm{ppm}$, respectively) for S_CK from Figure 1C and D as the initial measures of grain quality for scenarios with low and high FHB intensities. We then used these values to estimate the net cash income for S_CK as $N C I_{S C K}=Y l d \cdot(P-d c t)$ at grain prices $(P)$ of US\$118, US\$197, and US\$276/MT. Based on data from the U.S. Department of Agriculture, National Agricultural Statistics Service, these values were within the range of prices received for wheat grain between December 2013 and December 2015 across several of the states included in this study. For instance, using mean grain yield and test weight and the first quantiles for FDK and DON (10.5\% and $2.3 \mathrm{ppm}$, respectively), $N C I_{S_{-} C K}$ was estimated at low FHB intensity as US\$346.6/ha $([118-37.4] \times 4.3)$ at a grain price of US\$118/MT, US\$686.3/ha ([197 - 37.4] × 4.3) at US\$197/MT, and US\$1,026/ha $([276-37.4] \times 4.3)$ at US\$276/MT. In each case, US\$37.4/MT was subtracted from $P$ as the total price discount $(d c t)$ due to test weight below and DON and FDK above grain quality thresholds (Salgado et al. 2014, 2017). Similarly, $N C I_{S_{-} C K}$ was estimated for each grain price under high FHB intensity using the third quantiles of the distributions of FDK and DON as baseline measures of grain quality and applying the appropriate price discounts.

Baseline responses were adjusted to account for management program effects ( $\bar{D}$ and $\bar{C}$ values in Tables 5 to 7 and Fig. 2), price discounts were recalculated $\left(d c t^{*}\right)$ to reflect improvements in grain quality, and NCI was estimated for each management program as $\left.N C I_{M g m t}=Y l d^{*} \cdot\left(P-d c t^{*}\right)-\operatorname{Cost}_{p}\right)$. Yield and test weight were adjusted by adding $\bar{D}$ (for yield and test weight) to $4.3 \mathrm{MT} / \mathrm{ha}$ and $684 \mathrm{~kg} / \mathrm{m}^{3}$, respectively, whereas FDK and DON were adjusted by reducing the baseline values by the percent control $(\bar{C})$. Where appropriate, adjustments were made using $\bar{D}$ and $\bar{C}$ values at $\mathrm{IND}_{\mathrm{S}_{-} \mathrm{CK}}>10 \%$ and $\mathrm{IND}_{\mathrm{S}_{-} \mathrm{CK}} \leq 10 \%$ based on results from the analyses with $I_{\text {IND_CK }}$ as the moderator variable (Tables 5 to 7). However, for those management programs for which the effect of IND $_{\mathrm{S}_{\mathrm{CK}}}$ on $\bar{L}$ (and $\bar{C}$ ) was not statistically significant, the baseline values were adjusted based on the overall $\bar{D}$ and $\bar{C}$ values (from the analysis without the moderator [Table 4 and Fig. 2]). For scenarios in which the effect of a given management program was not statistically significant (i.e., $\bar{L}$ or $\bar{D}$ not significantly different from zero; Table 6), the baseline response was left unchanged. For instance, for MR_A, the baseline DON at low FHB intensity $(2.3 \mathrm{ppm})$ was reduced by $49 \%(\bar{C}$ in Table 7) to $1.2 \mathrm{ppm}[2.3 \times((100-\bar{C}) / 100)]$; baseline yield was increased from $4.3 \mathrm{MT} / \mathrm{ha}$ to $4.6 \mathrm{MT} / \mathrm{ha}(4.3+0.30$, the $\bar{D}$ value in Table 4); and FDK was reduced by $53.5 \%(\bar{C}$ in Table 6$)$ from 10.5 to $4.9 \%$. However, since MR_A did not have a significant effect on test weight at low FHB intensity, the baseline test weight value was left unchanged at $684 \mathrm{~kg} / \mathrm{m}^{3}$.

Using fungicide application costs ranging from $\$ 40$ to $\$ 96 \mathrm{ha}^{-1}$ ( Cost $_{p}$; product and application), $N C I_{M g m t}$ was estimated for each management program and then used to estimate the economic benefits (EB) depicted in Figure 4 as $N C I_{M g m t}-N C I_{S_{-} C K}$. Across all management programs, application costs, and grain prices, $\mathrm{EB}$ values were low, below US\$200, with low baseline FHB intensity (Fig. 4A, C, and E), and considerably higher at the higher FHB intensity (Fig. 4B, D, and F). At all grain prices tested, the EB was highest for programs in which an MS cultivar was used along with a prothioconazole + tebuconazole application and lowest for fungicide-only programs. At the high FHB intensity, EB decreased with delayed fungicide applications, particularly for programs with MS or S cultivars (Fig. 4B, D, and F). EB values were within a fairly narrow range across application timings and grain prices for programs with an MR cultivar. For instance, at any given grain price, the difference in EB between pairs of application timings, at a cost of $\$ 60 / \mathrm{ha}$, was less than US\$44/MT at high FHB intensity (Fig. 4F). Similarly, for management program with an MR cultivar plus a prothioconazole + 
tebuconazole application at \$60/ha, the difference in EB between the highest and lowest tested grain prices ranged from US $\$ 40.00 /$ MT to US\$53.00/MT. The corresponding differences were higher for management programs that include a prothioconazole + tebuconazole application to an MS cultivar, ranging from US\$85/MT to US\$118/MT.

\section{Discussion}

FHB continues to be difficult to control. This is partly because no cultivar is immune or highly resistant to FHB, the best fungicides alone are usually less than $60 \%$ effective, and practical limitations frequently prevent fungicides from being applied at the recommended growth stage (early anthesis). Here we present results from a multi-state study conducted to examine the integration of genetic resistance and fungicide application timing in an effort to develop management guidelines that are effective and at the same time robust to conditions experienced in the field. Our results showed that the benefits of integrating fungicide treatment and genetic resistance to manage FHB and DON were not greatly compromised by applying the fungicide 2 to 7 days after (referred to here as post-anthesis)

\section{Low baseline FHB index}

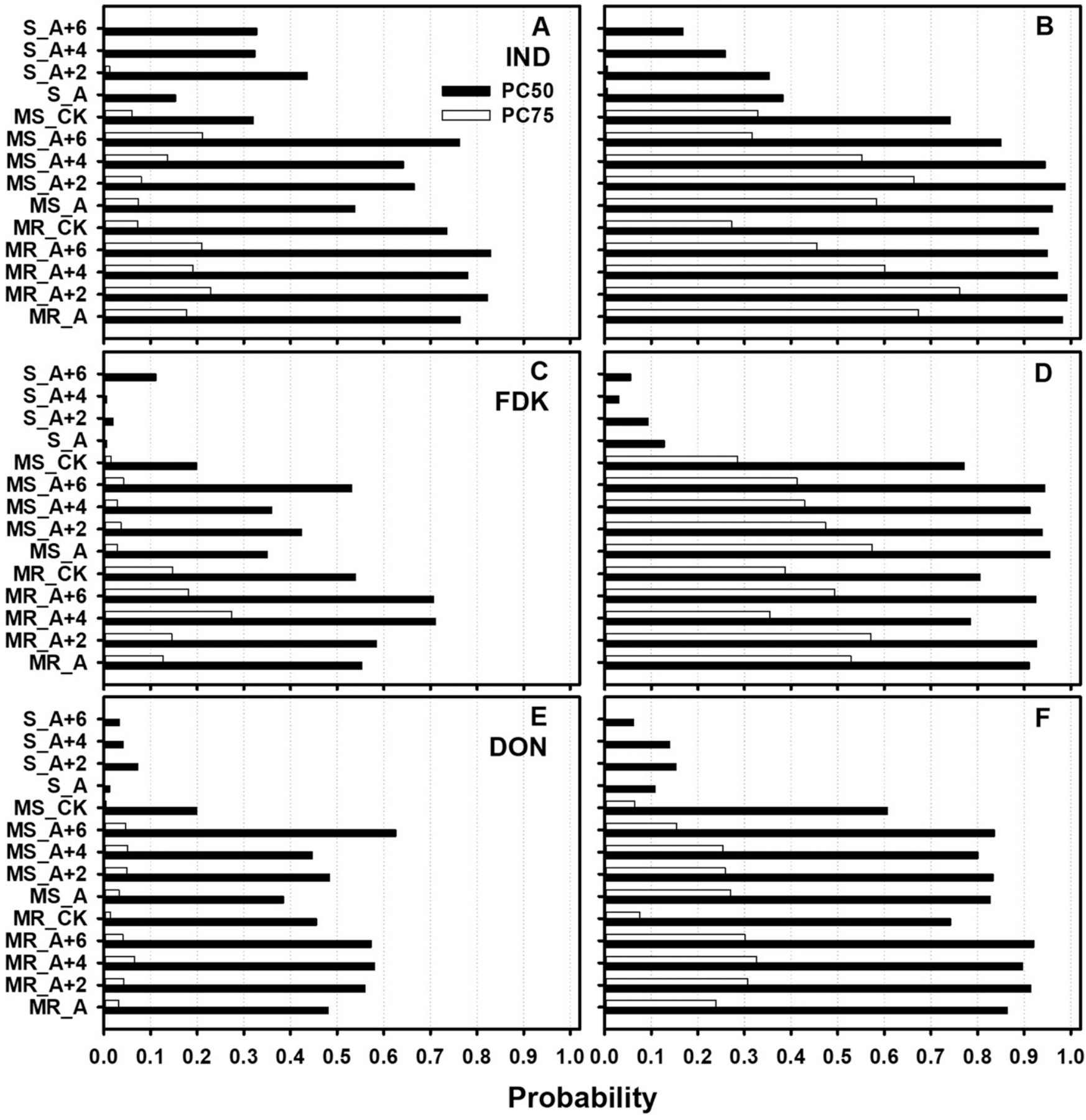

Fig. 3. Estimated probabilities of observing a 50 or $75 \%$ reduction in A and B, Fusarium head blight index (IND; percentage of diseased spikelets per spike); $\mathbf{C}$ and $\mathbf{D}$, Fusarium damaged kernels (FDK; percentage of visibly small, shriveled, and discolored kernels); and $\mathbf{E}$ and $\mathbf{F}$, deoxynivalenol (DON) contamination of harvested grain for different fungicide treatment by cultivar resistance class combinations relative to a nontreated susceptible check, respectively, in trials with low $(\mathbf{A}, \mathbf{C}$, and $\mathbf{E})$ and high $(\mathbf{B}, \mathbf{D}$, and $\mathbf{F})$ baseline levels of IND. Management combinations consisted of the application of the fungicide prothioconazole + tebuconazole at $50 \%$ early anthesis, or at 2, 4, or 6 days after $50 \%$ early anthesis (A, $A+2, A+4$, or $A+6$, respectively) to moderately resistant (MR), moderately susceptible (MS), or susceptible (S) wheat cultivars. Probability values were calculated using the estimated mean log response ratios and their between study variances in Tables 5, 6, and 7. 
instead of at $50 \%$ early anthesis. Based on a quantitative synthesis of data from integrated management trials similar to those in this investigation, Willyerd et al. (2012) reported that a single anthesis application of prothioconazole + tebuconazole to an MR cultivar (equivalent to our MR_A) reduced mean IND and DON by $76 \%$ (CI: 67 to 82 ) and $71 \%$ (CI: 64 to 77 ), respectively, relative to the nontreated susceptible check (our S_CK). These levels of efficacy are comparable to the 64 to $82 \%$ observed for IND and the 56 to $70 \%$ for DON with post-anthesis applications of the same fungicide to an MR cultivar in this study. The integrated approach with a postanthesis application was up to 11 and $9 \%$ more effective than resistance alone for IND and DON, respectively, and up to $38 \%$ more effective than fungicide application alone. Our finding also showed that the efficacy of post-anthesis fungicide applications was consistent across cultivars with different levels of genetic resistance to FHB, with treatments applied 2 to 7 days after $50 \%$ early anthesis being just as, and occasionally, more effective in terms of percent IND and DON control than treatments applied at anthesis.

The fact that the integration of multiple management strategies was generally more effective against IND and DON than a single strategy has been well documented and discussed (McMullen et al. 2008; Wegulo et al. 2011; Willyerd et al. 2012; Yuen and

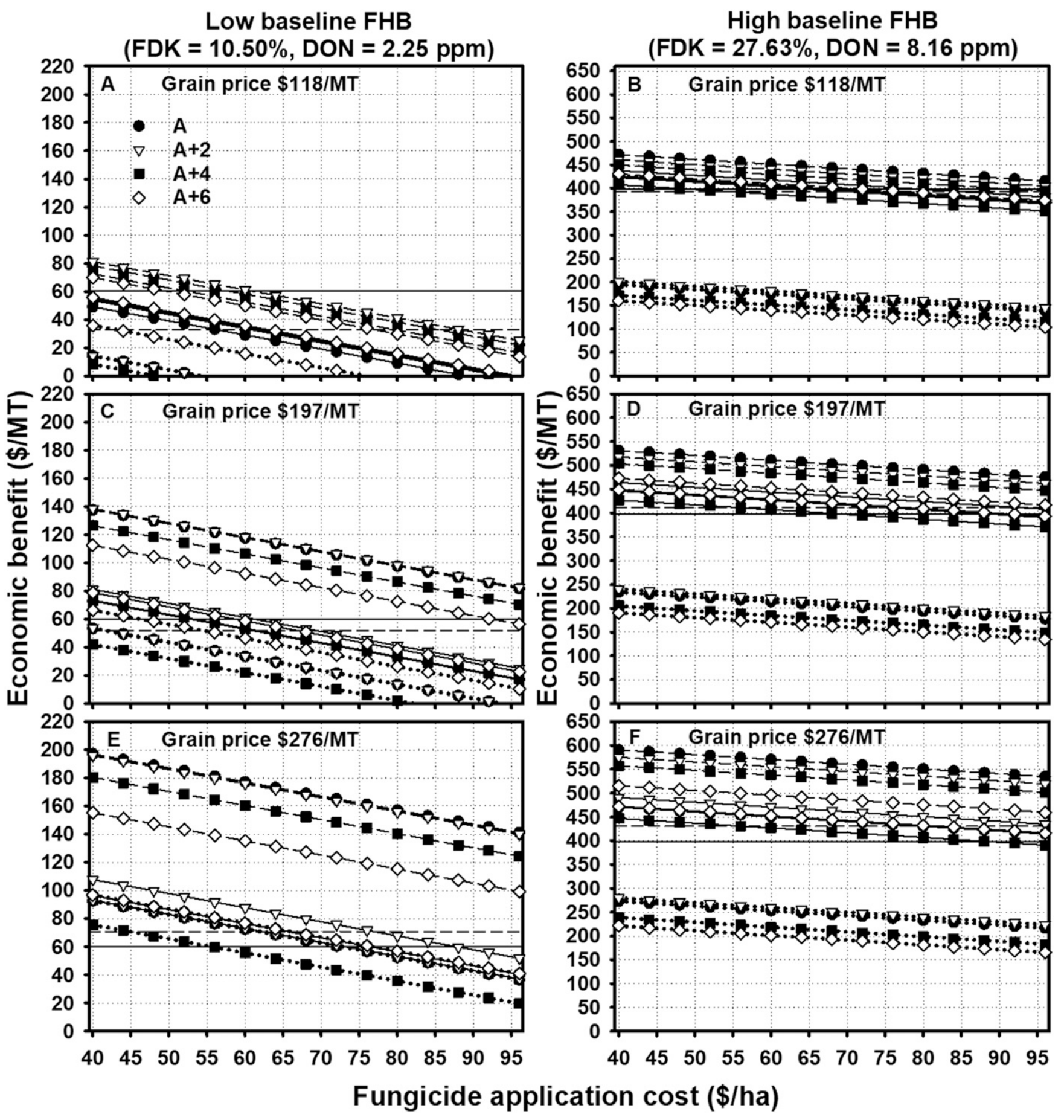

Fig. 4. Estimated economic benefit (EB) at different grain prices and fungicide application costs for fungicide treatment by cultivar resistance class combinations used to manage Fusarium head blight at low $(\mathbf{A}, \mathbf{C}$, and $\mathbf{E})$ and high $(\mathbf{B}, \mathbf{D}$, and $\mathbf{F})$ baseline levels of disease. Management combinations consisted of the application of the fungicide prothioconazole + tebuconazole at $50 \%$ early anthesis, or at 2, 4, or 6 days after $50 \%$ early anthesis (A, A+2, A+4, or A+6, respectively, represented by different symbols) to moderately resistant (solid lines), moderately susceptible (broken lines), or susceptible (dotted lines) wheat cultivars. Nontreated moderately resistant and nontreated moderately susceptible are represented by solid and broken reference lines without symbols, respectively. 
Schoneweis 2007). However, IND and DON results alone cannot be used as measures of the effects of fungicide, genetic resistance, or other management strategies on other components of FHB, grain quality, or yield. Even though these responses are interrelated (Dill-Macky and Jones 2000; Jones 2000; Madden and Paul 2009; Paul et al. 2005; Salgado et al. 2015; Snijders and Perkowski 1990), the relationships are highly variable and may be influenced by environment- and cultivar-related factors (Boyacioglu et al. 1992; Cowger et al. 2009, 2016; Culler et al. 2007; Paul et al. 2005; Sneller et al. 2012). Although INC and IND are functionally related (Paul et al. 2006), they are often quantified separately and used as measures of Type I and Type II resistance (resistance to initial infection and fungal spread within the spike [Bal and Shaner 1994, 2004; Schroeder and Christensen 1963]), respectively. Similarly, Sneller et al. (2012) quantified grain colonization (through real-time polymerase chain reaction analyses) and DON separately as measures of resistance to kernel infection and toxin accumulation, respectively. By extension, in this study, separate meta-analytical models were fitted to each of the aforementioned responses to more directly quantify management program effects on measures of spike infection and colonization, and grain colonization, contamination with DON, yield, and test weight. Moreover, since each response contributes to a separate component of the overall economic impact of FHB (McMullen et al. 1997; Nganje et al. 2004), we opted to analyze them separately to generate estimates that can be used for future assessments of the economic benefits of FHB management programs.

Noteworthy differences were evident in terms of the magnitude of the integrated management effects across the four measures of FHB. Between the two in-field responses examined, efficacy was greater against IND than INC, whereas between the two postharvest measures of grain quality, the integrated approach was more efficacious against FDK than against DON. These results seem to suggest that under the conditions of this study, the integrated management programs were more effective at reducing colonization and spread within the spike and grain than at reducing initial infections and toxin accumulation. However, the relatively inferior performance of management strategies against DON compared with IND may be attributed, at least in part, to the fact that the DON content of grain may change in response to post-anthesis weather conditions (Cowger and Arellano 2013; Cowger et al. 2009; Culler et al. 2007). Under certain environmental conditions (for instance, extended periods of preharvest rainfall), DON levels may increase well after a fungicide treatment is applied. Post-anthesis moisture also affects FDK (Cowger and Arellano 2013; Cowger et al. 2009), which may in part explain the observed inferior efficacy of fungicide treatments against this response compared with efficacy against IND, particularly for the more susceptible cultivars (likely with low levels of resistance to kernel infection [Sneller et al. 2012]).

Given that plots in most of the experiments were spray-inoculated at anthesis, one might have expected applications made 2 to 7 days after inoculation to be less effective against INC than those made at anthesis. Instead, as was observed for IND, FDK, and DON, post-anthesis applications were just as effective as or more effective than the anthesis application against INC. However, spray inoculation did seem to play a role in determining INC, with this being the only measure of FHB for which the effect of baseline IND (used here as a surrogate for environmental favorability) was not statistically significant for 11 of the 14 management programs. For all other FHB-related responses, efficacy was generally higher (and sometimes considerably so) under more favorable conditions, but trends in terms of the most efficacious management program were similar to those discussed above for the general situation. Using mean DON as a surrogate for conditions favorable for FHB, Cowger et al. (2016) also reported greater treatment effects on DON under favorable conditions and inferred similar effects on FDK, IND, and kernel infection based on correlations. For INC, we hypothesize that the water used for inoculation was sufficient for spikes to become infected, making this process less dependent on the local environmental conditions than later colonization of the spike and grain. Another interesting finding was that on MR cultivars, the gain in efficacy with a fungicide application in terms of percent reduction relative to the nontreated susceptible check was highest for INC, followed by IND and DON, and lowest for FDK. These results could be related to the complex nature of resistance to FHB, as the MR cultivars used in the study may have had higher levels of resistance to kernel infection, fungal spread within the spike, and DON accumulation than resistance to initial infections of the spike (Sneller, personal communication).

Among the most interesting findings were the effects of management programs and baseline IND on grain yield response. Under the conditions of this study, yield response to most of the tested management programs (13 of 14) was not influenced by baseline IND. This is dissimilar to the results in Paul et al. (2010), which showed that wheat yield responses to DMI fungicides, both in relative and absolute terms, were lower at 5\% IND than at 30\% (comparable to the overall mean [28\%] for the high IND_CK category in this study). Similarly, Cowger et al. (2016) showed greater effects of fungicide treatments and cultivar resistance on grain yield in high-DON environments than in low-DON environments. Although the effect of IND_CK on yield response $(\bar{D})$ to management programs was not statistically significant $(P>0.05)$ in our study, there was a consistent trend toward numerically higher $\bar{D}$ values in trials with IND_CK > $10 \%$ than in those with IND_CK $\leq 10 \%$. The lack of significant IND_CK effect on yield response could be attributed to the relatively smaller sample size, and correspondingly, high variability, for each management program in each category of the moderator variable (5 to 12 trials in the low IND_CK category and 11 to 17 trials in the high IND_CK category).

Reducing the mean proportion of spikes (INC) and spikelets (IND) diseased and damage to the grain (FDK) contributed to increases in test weight, as the most effective programs against measures of FHB also resulted in the highest test weights. This was expected as test weight is a measure of grain mass per unit volume, and a healthier grain sample would have a higher mass than a more diseased sample at a fixed volume. The magnitude and significance of the management program effects on test weight were greater under conditions that were more favorable for FHB than under conditions that were less favorable. These results are consistent with those reported by Paul et al. (2010) and Cowger et al. (2016). However, an increase in the proportion of healthy and plump kernels in management programs including an MR cultivar was not sufficient to result in proportional increases in grain yield in terms of weight per unit area. We attribute this to the fact that the overall mean yield (average across trials) was lower for the MR cultivars than the MS and S cultivars. In other words, we believe that the response was due to inherent differences in yield among the cultivars in the S, MS, and MR resistance classes. For instance, even with higher overall mean FHB index, the nontreated checks for the susceptible and moderately susceptible $\left(\mathrm{S}_{-}\right.$ CK and MS_CK) cultivars had mean yields of 4,335 and 4,603 $\mathrm{kg} / \mathrm{ha}$, respectively, compared with $4,226 \mathrm{~kg} / \mathrm{ha}$ for the nontreated check of the moderately resistant cultivar (MR_CK). This combined with the fact that the yield gain with a fungicide treatment was higher within the susceptible (a mean increase for 386 to $498 \mathrm{~kg} / \mathrm{ha}$ for Streated over S_CK) and moderately susceptible (301 to $513 \mathrm{~kg} / \mathrm{ha}$ increase for MS-treated over MS_CK) classes than within the moderately resistant class ( 255 to $305 \mathrm{~kg} / \mathrm{ha}$ increase for MR-treated over MR_CK) likely resulted in lower overall $\bar{D}$ values for programs with the MR cultivar.

Understandably, differences in terms of efficacy against measures of FHB and grain quality affected the projected future effects and the estimated economic benefits (EB) of the management programs evaluated in this study. The more effective integrated management programs (those with MR or MS cultivars plus a fungicide application) were the most likely to result in at least a 50 or $70 \%$ control of IND, FDK, or DON in future trials and were also the most economically beneficial. In addition, both the estimated probability of future efficacy and EB of any given program were higher under moderate-to-high FHB intensity than under low intensity within the range of FHB levels observed in this study. These general results 
were not surprising, as the probability of a response of a given magnitude was estimated in this study as a direct function of mean efficacy, which by definition was highest for the most effective programs. With respect to $\mathrm{EB}$, reductions in price discounts due to improved grain quality (lower FDK and DON and higher test weight) resulted in higher prices being received for grain (lower price discounts), and this coupled with higher grain production per unit area led to the integrated programs being more economically beneficial than fungicide treatments alone. However, differences between these programs in terms of the magnitude of the EB clearly reflected the relative contributions of yield, grain quality, and grain market price to the overall EB of FHB management.

Although programs with MR cultivars were among the most effective at improving grain quality, and consequently, reducing price discounts, these programs were less economically beneficial than those with MS cultivars at high grain prices. The latter group of programs had slightly lower efficacy than the former in terms of FDK and DON reduction, but resulted in considerably higher mean yield responses. At the highest grain prices tested, higher grain yields for programs with MS cultivars compared with those with MR cultivars compensated for slightly poorer grain quality, leading to the former being more economically beneficial. Salgado et al. (2014) also showed that, although integrated management programs were effective at reducing FHB and improving grain quality, this superior disease and toxin control did not always translate into the highest EB when the MR cultivar had a lower yield potential than the $\mathrm{S}$ cultivar. In the economic model used in this study, the price penalty for a program having lower grain quality represents a smaller proportion of the grain price at higher prices than at low prices, and the monetary value of a high yield response is a larger proportion of the price than a marginal price discount. For instance, with MS_A+2 at a grain price of US\$118/ MT, a total price discount of US\$18/MT for DON and FDK above thresholds represented $15 \%$ of the grain price, whereas at US\$276/ MT, the same price discount represented only approximately $5 \%$ of the grain price. On the other hand, the monetary value of the average difference in yield between programs with a relatively lowyielding MR cultivar and those with higher-yielding MS cultivar, $0.35 \mathrm{MT} / \mathrm{ha}$, was worth $\$ 97.35 /$ ha at US\$276/MT and was equivalent to $35 \%$ of the price.

In this study, we have demonstrated the benefit of combining genetic resistance with a fungicide treatment to manage FHB, even if that treatment has to be applied a few days after $50 \%$ early anthesis. We also demonstrated the effects of the complex interplay among efficacy, yield response, and grain price on the economic benefit of FHB management. Cowger et al. (2016) also demonstrated and discussed this complexity, with general conclusions comparable to those highlighted in our investigation; to be economically beneficial, an FHB management program has to not only reduce disease intensity, but also improve grain yield and quality to levels high enough to offset management cost at a given grain price. Based on our results, we concluded that under less favorable conditions for FHB (defined here as approximately $5 \%$ for IND, $10.5 \%$ for FDK, and $2.25 \mathrm{ppm}$ for DON in the nontreated susceptible check), a prothioconazole + tebuconazole application to an S cultivar was not economically beneficial at the lowest, and more realistic, grain price of US\$118/MT (US $\$ 3.00$ per bushel), and only marginally beneficial when applied to an MR or MS cultivar (the integrated approach). However, at that same grain price, under more FHB-favorable conditions (defined as approximately $28 \%$ IND, $28 \%$ FDK, and 8 ppm DON), all programs were economically beneficial. Programs with a fungicide application alone benefitted from marginal improvements in grain quality, but most importantly from the fact that the susceptible cultivars in this study were relatively high-yielding. Programs with MR cultivars benefitted from superior overall efficacy and good yield responses, whereas those with MS cultivars benefitted from superior yield and very good quality responses, leading to the two groups of programs having comparable economic benefit at a grain price of US $\$ 118 / \mathrm{MT}$.

It is important to note that the results and conclusions presented above are based on FHB and associated quality responses within manageable ranges and makes the assumption that grain is not entirely rejected at the buying point when it met the baseline quality (DON, FDK, and test weight) levels used here. Depending on the grain market, how widespread the disease is in a given year and region, and the availability of grain buyers to source healthy or less damaged grain, grain may be rejected even after the most effective managed programs are implemented. However, because FHB epidemics are heavily dependent on weather and the heterogeneous nature of disease development in commercial fields, and thanks to progress made developing resistant cultivars (McMullen et al. 2012), naturally incited FHB in the U.S. is often within manageable ranges under field conditions; this will likely lead to grain quality traits within marketable ranges following the implementation of the best integrated management programs as identified in this study.

\section{Acknowledgments}

Special thanks to W. Bardall, M. Wallhead, and the Snyder from crew for assisting with the establishment and maintenance of plots in Wooster, OH; L. Siler and E. Olson for establishment and maintenance of plots in East Lansing, MI; K. Ames, M. Johnson, B. Mansfield, and J. Pike for assistance with field trials in Illinois; and P. Stachowski and R. J. Richtmyer for establishment, maintenance, and spraying of plots in Aurora, NY.

\section{Literature Cited}

Andersen, K. F., Morris, L., Derksen, R. C., Madden, L. V., and Paul, P. A. 2014 Rainfastness of prothioconazole + tebuconazole for Fusarium head blight and deoxynivalenol management in soft red winter wheat. Plant Dis. 98:1398-1406

Bal, G. H., and Shaner, G. 1994. Scab of wheat: prospects for control. Plant Dis. 78:760-766.

Bal, G. H., and Shaner, G. 2004. Management and resistance in wheat and barley to Fusarium head blight. Annu. Rev. Phytopathol. 42:135-161.

Barker, D., Culman, S., Dorrance, A., Fulton, J., Haden, R., Lentz, E., Lindsey, A., Lindsey, L., Loux, M., McCoy, E., Michel, A., Noel, J., Paul, P., Sulc, M., Thomison, P., Tilmon, K., and Witter, J. 2017. Ohio Agronomy Guide, 15th Edition. The Ohio State University. Bulletin 472.

Boyacioglu, D., Hettiarachchy, N. S., and Stack, R. W. 1992. Effect of three systemic fungicides on deoxynivalenol (vomitoxin) production by Fusarium graminearum in wheat. Can. J. Plant Sci. 72:93-101.

Bradley, C. A., Adee, E. A., Ebelhar, S. A., Dill-Macky, R., Wiersma, J. J., Grybauskas, A. P., Kirk, W. W., McMullen, M. P., Halley, S., Milus, E. A., Osborne, L. E., Ruden, K. R., and Young, B. G. 2010. Multi-state uniform fungicide evaluations for control of Fusarium head blight and associated mycotoxins. Page 74 in: Proc. 2010 National Fusarium Head Blight Forum. S. Canty, A. Clark, A. Anderson-Scully, D. Ellis, and D. Van Sanford, eds. Milwaukee, WI.

Bradley, C. A., Adee, E. A., Ebelhar, S. A., Grybauskas, A. P., Hollingsworth, C. R., Kirk, W. W., McMullen, M. P., Milus, E. A., Osborne, L. E., Ruden, K. R., and Young, B. G. 2009. Application timings of Caramba and Prosaro foliar fungicides for management of FHB and DON. Page 34 in: Proc. 2009 National Fusarium Head Blight Forum. S. Canty, A. Clark, J. Mundell, E. Walton, D. Ellis, and D. Van Sanford, eds. Orlando, FL.

Conley, S., Roth, A., Gaska, J., and Smith, D. 2014. Wisconsin Winter Wheat Performance Tests 2014. UW-Extension Publication A3868.

Cowger, C., and Arellano, C. 2013. Fusarium graminearum infection and deoxynivalenol concentrations during development of wheat spikes. Phytopathology 103:460-471.

Cowger, C., Patton-Ozkurt, J., Brown-Guedira, G., and Perugini, L. 2009. Postanthesis moisture increased Fusarium head blight and deoxynivalenol levels in North Carolina winter wheat field experiment. Phytopathology 99:320-327.

Cowger, C., Weisz, R., Arellano, C., and Murphy, P. 2016. Profitability of integrated management of Fusarium head blight in North Carolina winter wheat. Phytopathology 106:814-823.

Culler, M. D., Miller-Garvin, J. E., and Dill-Macky, R. 2007. Effect of extended irrigation and host resistance on deoxynivalenol accumulation in Fusariuminfected wheat. Plant Dis. 91:1464-1472.

D’Angelo, D. L., Bradley, C. A., Ames, K. A., Willyerd, K. T., Madden, L. V., and Paul, P. A. 2014. Efficacy of fungicide applications during and after anthesis against Fusarium head blight and deoxynivalenol in soft red winter wheat. Plant Dis. 98:1387-1397.

Dill-Macky, R., and Jones, R. K. 2000. The effect of previous crop residues and tillage on Fusarium head blight of wheat. Plant Dis. 84:71-76.

Hedges, L. V., Gurevitch, J., and Curtis, P. S. 1999. The meta-analysis of response ratios in experimental ecology. Ecology 80:1150-1156.

Jones, R. K. 2000. Assessments of Fusarium head blight of wheat and barley in response to fungicide treatment. Plant Dis. 84:1021-1030.

Littell, R. C., Milliken, G. A., Stroup, W. W., Wolfinger, R. D., and Schabenberger, O. 2006. SAS System for Mixed Models. SAS Institute, Cary, NC. 
Madden, L. V., and Paul, P. A. 2009. Assessing heterogeneity in the relationship between wheat yield and Fusarium head blight intensity using randomcoefficient mixed models. Phytopathology 99:850-860.

Madden, L. V., and Paul, P. A. 2011. Meta-analysis for evidence synthesis in plant pathology: An overview. Phytopathology 101:16-30.

Madden, L. V., Piepho, H.-P., and Paul, P. A. 2016. Statistical models and methods for network meta-analysis. Phytopathology 106:792-806.

Mansfield, C., and Hawkins, S. 1992. Agronomy Guide - Wheat Production and Fertilization in Indiana. Extension Bulletin (AY-244). West Lafayette, IN.

McMullen, M., Bergstrom, G., De Wolf, E., Dill-Macky, R., Hershman, D., Shaner, G., and Van Sanford, D. 2012. A Unified Effort to Fight an Enemy of Wheat and Barley: Fusarium Head Blight. Plant Dis. 96:1712-1728.

McMullen, M., Halley, S., Schatz, B., Meyer, S., Jordahl, J., and Ransom, J. 2008. Integrated strategies for Fusarium head blight management in the United States. Cereal Res. Commun. 36 (Supplement 6):563-568.

McMullen, M., Jones, R., and Gallenberg, D. 1997. Scab of wheat and barley: A reemerging disease of devastating impact. Plant Dis. 81:1340-1348.

Mesterházy, Á., Bartók, T., and Lamper, C. 2003. Influence of wheat cultivar, species of Fusarium, and isolate aggressiveness on the efficacy of fungicides for control of Fusarium head blight. Plant Dis. 87:1107-1115.

Mills, K. B., Salgado, J. D., Madden, L. V., and Paul, P. A. 2017. The spray before the storm: assessing the rainfastness of Caramba for control of Fusarium head blight. Page 16 in: Proc. 2017 National Fusarium Head Blight Forum. S. Canty, B. Wiermer, and D. Van Sanford, eds. Milwaukee, WI.

Nafziger, E., ed. 2009. Illinois Agronomy Handbook, 24th Ed. University of Illinois Extension, Urbana, IL.

Nganje, W. E., Bangsund, D. A., Leistritz, F. L., Wilson, W. W., and Tiapo, N. M. 2004. Regional economic impacts of Fusarium head blight of wheat and barley. Rev. Agric. Econ. 26:332-347.

Paul, P. A., Bradley, C. A., Madden, L. V., Dalla Lana, F., Bergstrom, G. C., DillMacky, R., Wise, K., Esker, P. D., McMullen, M. P., Grybauskas, A., Kirk, W. W., Milus, E., and Ruden, K. 2018. Effects of pre- and post-anthesis applications of demethylation inhibitor fungicides on Fusarium head blight and deoxynivalenol in spring and winter wheat. Plant Dis. doi:10.1094/PDIS03-18-0466-RE

Paul, P. A., Lipps, P. E., Hershman, D. E., McMullen, M. P., Draper, M. A., and Madden, L. V. 2007. A quantitative review of tebuconazole effect on Fusarium head blight and deoxynivalenol content in wheat. Phytopathology 97:211-220.

Paul, P. A., Lipps, P. E., Hershman, D. E., McMullen, M. P., Draper, M. A., and Madden, L. V. 2008. Efficacy of triazole-based fungicides for Fusarium head blight and deoxynivalenol control in wheat: A multivariate meta-analysis. Phytopathology 98:999-1011.

Paul, P. A., Lipps, P. E., and Madden, L. V. 2005. Relationship between visual estimates of Fusarium head blight intensity and deoxynivalenol accumulation in harvested wheat grain: A meta-analysis. Phytopathology 95:1225-1236.

Paul, P. A., Lipps, P. E., and Madden, L. V. 2006. Meta-analysis of regression coefficients for the relationship between Fusarium head blight and deoxynivalenol content of wheat. Phytopathology 96:951-961.
Paul, P. A., McMullen, M. P., Hershman, D. E., and Madden, L. V. 2010. Metaanalysis of the effects of triazole-based fungicides on wheat yield and test weight as influenced by Fusarium head blight intensity. Phytopathology 100:160-171.

Salgado, J. D., Lindsey, L., and Paul, P. A. 2017. Effects of row spacing and nitrogen rate on wheat grain yield and profitability as influenced by diseases. Plant Dis. 101:1998-2011.

Salgado, J. D., Madden, L. V., and Paul, P. A. 2014. Efficacy and economics of integrating in-field and harvesting strategies to manage Fusarium head blight of wheat. Plant Dis. 98:1407-1421.

Salgado, J. D., Madden, L. V., and Paul, P. A. 2015. Quantifying the effects of Fusarium head blight on grain yield and test weight in soft red winter wheat. Phytopathology 105:295-306.

Salgado, J. D., Wallhead, M., Madden, L. V., and Paul, P. A. 2011. Grain harvesting strategies to minimize grain quality losses due to Fusarium head blight in wheat. Plant Dis. 95:1448-1457.

Schroeder, H. W., and Christensen, J. J. 1963. Factors affecting the resistance of wheat to scab caused by Gibberella zeae. Phytopathology 53:831-838.

Shah, D. A., De Wolf, E. D., Paul, P. A., and Madden, L. V. 2014. Predicting Fusarium head blight epidemics with boosted regression trees. Phytopathology 104:702-714.

Shah, D. A., Molineros, J. E., Paul, P. A., Willyerd, K. T., Madden, L. V., and De Wolf, E. D. 2013. Predicting Fusarium head blight epidemics with weatherdriven pre- and post-anthesis logistic regression models. Phytopathology 103: 906-919.

Sneller, C., Guttieri, M., Paul, P., Costa, J., and Jackwood, R. 2012. Variation for resistance to kernel infection and toxin accumulation in winter wheat infected with Fusarium graminearum. Phytopathology 102:306-314.

Snijders, C. H. A., and Perkowski, J. 1990. Effects of head blight caused by Fusarium culmorum on toxin content and weight of wheat kernels. Phytopathology 80:566-570.

Stack, R. W., and McMullen, M. P. 1998. A visual Scale to estimate severity of Fusarium head blight in wheat. NDSU Extension Service: Small Grains Publications. Online Publication/PP-1095.

van Houwelingen, H. C., Arends, L. R., and Stijnen, T. 2002. Advanced methods in meta-analysis: Multivariate approach and meta-regression. Stat. Med. 21:589-624.

Wegulo, S. N., Bockus, W. W., Nopsa, J. H., De Wolf, E. D., Eskridge, K. M. Peiris, K. H. S., and Dowell, F. E. 2011. Effects of integrating cultivar resistance and fungicide application on Fusarium head blight and deoxynivalenol in winter wheat. Plant Dis. 95:554-560.

Willyerd, K. T., Li, C., Madden, L. V., Bradley, C. A., Bergstrom, G. C., Sweets, L. E., McMullen, M., Ransom, J. K., Grybauskas, A., Osborne, L., Wegulo, S. N., Hershman, D. E., Wise, K., Bockus, W. W., Groth, D., Dill-Macky, R., Milus, E., Esker, P. D., Waxman, K. D., Adee, E. A., Ebelhar, S. E., Young, B. G., and Paul, P. A. 2012. Efficacy and stability of integrating fungicide and cultivar resistance to manage Fusarium head blight and deoxynivalenol in wheat. Plant Dis. 96:957-967.

Yuen, G. Y., and Schoneweis, S. D. 2007. Strategies for managing Fusarium head blight and deoxynivalenol accumulation in wheat. Int. J. Food Microbiol. 119: 126-130. 